\title{
Microscopic Conductivity of Lattice Fermions at Equilibrium - Part II: Interacting Particles
}

\author{
Jean-Bernard Bru Walter de Siqueira Pedra
}

September 24, 2015

\begin{abstract}
We apply Lieb-Robinson bounds for multi-commutators we recently derived [BP3] to study the (possibly non-linear) response of interacting fermions at thermal equilibrium to perturbations of the external electromagnetic field. This analysis leads to an extension of the results for quasi-free fermions of [BPH1, BPH2] to fermion systems on the lattice with shortrange interactions. More precisely, we investigate entropy production and charge transport properties of non-autonomous $C^{*}$-dynamical systems associated with interacting lattice fermions within bounded static potentials and in presence of an electric field that is time- and space-dependent. We verify the 1st law of thermodynamics for the heat production of the system under consideration. In linear response theory, the latter is related with $\mathrm{Ohm}$ and Joule's laws. These laws are proven here to hold at the microscopic scale, uniformly with respect to the size of the (microscopic) region where the electric field is applied. An important outcome is the extension of the notion of conductivity measures to interacting fermions.
\end{abstract}

Keywords: Disordered systems; transport processes; conductivity measure AMSC: 82C70, 82C44, 82C20

\section{Contents}

1 Introduction 
$2 C^{*}$-Dynamical Systems for Interacting Fermions 4

2.1 Free Fermions within Electromagnetic Fields . . . . . . . . . . 4

2.2 CAR $C^{*}$-Algebra of the Infinite Lattice . . . . . . . . . . 7

2.3 Banach Space of Short-Range Interactions . . . . . . . . . . 7

2.4 Unperturbed Dynamics of Interacting Fermions . . . . . . . . . . 9

2.5 Dynamics Driven by Time-Dependent Electromagnetic Fields . . 11

2.6 Time-Dependent State of the System . . . . . . . . . . . . . . 13

3 Heat Production and Current Linear Response 14

3.1 Heat Production . . . . . . . . . . . . . . . . . . 14

3.2 Charge Transport Coefficients . . . . . . . . . . . 20

3.3 Microscopic Ohm's Law . . . . . . . . . . . . . . . . 25

3.4 Microscopic Joule's Law . . . . . . . . . . . . . . . . . . . 28

\section{Introduction}

The present paper belongs to a succession of works on Ohm and Joule's laws starting with [BPH1, BPH2, BPH3, BPH4]. These papers give a complete and mathematically rigorous derivation (at least in the $\mathrm{AC}$-regime) of the phenomenon of linear conductivity from microscopic quantum dynamics and first principles of thermodynamics, only. Their results lead, in particular, to a physical picture of the microscopic origin of Ohm and Joule's laws based on a notion of "quantum viscosity" for currents, highlighting the role of so-called diamagnetic and paramagnetic currents. However, a drawback of our previous studies [BPH1, BPH2, $\mathrm{BPH} 3, \mathrm{BPH} 4]$ is their restriction to non-interacting fermions in disordered media. Indeed, it is believed in theoretical physics that electric resistance of conductors should also result from interactions between charge carriers, and not only from the presence of inhomogenities (impurities).

Therefore, we aim to extend the results of [BPH1, BPH2, BPH3, BPH4] to fermion systems with interactions, i.e., to rigorously derive, at least in the $\mathrm{AC}-$ regime, Ohm and Joule's laws for interacting systems. See also [BP2] for a historical perspective of this subject. The present paper is a first step in this direction, extending all results of [BPH1, BPH2] to fermion systems on the lattice with short-range interactions and bounded static potentials:

- We investigate the heat production for some non-autonomous $C^{*}$-dynamical systems on the CAR $C^{*}$-algebra of cubic infinite lattices of any di- 
mension with respect to (w.r.t.) completely passive states. Such states implement the 2nd law of thermodynamics and are identified here with the (thermal) equilibrium states of the system. The (non-autonomous) dynamics is generated by a short-range interaction between particles, a bounded static potential, and next neighbor hoppings in presence of an electromagnetic field that is time- and space-dependent. In particular, we verify the 1st law of thermodynamics for the system under consideration.

- We next derive Ohm and Joule's laws at the microscopic scale, an unexpected $[\mathrm{F}]$ property experimentally verified in 2012 at the atomic scale [W]. More precisely, we show that, at weak external electric fields, uniformly w.r.t. the size of the region where the electric field is applied, the current density response is linear and the heat production density is quadratic in the strength of the applied field. We introduce the notion of conductivity measures for interacting fermions, similar to [KLM, KM1, KM2] and [BPH2, BPH3, BPH4] in the non-interacting case. Note however that the detailed structure of the conductivity measure is not studied, yet. It depends, of course, on the system under consideration.

Note that in [BPH2, Section 3.5] so-called microscopic conductivity distributions are defined from conductivity measures. Exactly the same construction, at least for time-reversal invariant models, could be done here and we refrain from doing it again. The same remark can be done for the derivation of Joule's law in its original formulation, see [BPH2, Section 4.5] for more details.

Like in [BPH1, BPH2], all estimates we get are uniform w.r.t. the size of the region with non-vanishing electric fields. This is possible because we prove in [BP3, Corollary 3.10] the tree-decay bounds on multi-commutators of [BPH1, Corollary 4.3] for the case of interacting fermions. These bounds are a pivotal ingredient in [BPH1, BPH2, BPH3, BPH4] and were derived in [BPH1, Section 4] by explicit computations using the fact that the fermion system was noninteracting. For interacting systems, we use in [BP3] Lieb-Robinson bounds for multi-commutators together with combinatorics of maximally connected graphs (tree expansions) to prove them. Note additionally that Lieb-Robinson bounds for multi-commutators also enter in a decisive way in the proof of the 1st law of thermodynamics for the case of interacting particles, see for instance Theorem 3.2 (i) and Remark 3.3.

As discussed in [BP3], this method requires short-range interactions. The short-range property is defined via the finiteness of a convenient norm for interactions: This norm ensures that the interaction is sufficiently weak at large 
distances. The set of such short-range interactions form a whole Banach space $\mathcal{W}$ w.r.t. this norm. The space $\mathcal{W}$ includes density-density interactions resulting from the second quantization of two-body interactions defined via a real-valued and summable (in a convenient sense) function $v(r):[0, \infty) \rightarrow \mathbb{R}$. Considering fermions with spins $\uparrow$ or $\downarrow$, our setting includes for instance the celebrated Hubbard model (and any other system with finite range interactions) or models with Yukawa-type potentials, but the Coulomb potential is excluded because it is not summable in space.

Our main assertions are Theorems 3.2-3.4 and 3.6-3.8. This paper is organized as follows:

- Section 2 precisely formulates our setting. We define in particular a Banach space of short-range interactions.

- Section 3 extends all results of [BPH1, BPH2] to fermion systems within bounded static potentials and with interactions decaying fast enough in space. Observe that, in contrast to [BPH2], we generally do not have timereversal invariance. This yields some (straightforward) adaptations of formulations of results and arguments.

\section{Notation 1.1}

To simplify notation, we denote by $D$ any generic positive and finite constant. These constants do not need to be the same from one statement to another. A norm on a generic vector space $\mathcal{X}$ is denoted by $\|\cdot\|_{\mathcal{X}}$ and the identity map of $\mathcal{X}$ by $1_{\mathcal{X}}$.

\section{$2 C^{*}$-Dynamical Systems for Interacting Fermions}

\subsection{Free Fermions within Electromagnetic Fields}

The host material for conducting fermions is assumed to be a cubic crystal represented by the $d$-dimensional cubic lattice $\mathfrak{L}:=\mathbb{Z}^{d}(d \in \mathbb{N})$.

Disorder in the crystal will be modeled by a random variable with distribution $\mathfrak{a}_{\Omega}$ taking values in the measurable space $\left(\Omega, \mathfrak{A}_{\Omega}\right)$, i.e., $\left(\Omega, \mathfrak{A}_{\Omega}, \mathfrak{a}_{\Omega}\right)$ is a probability space. Let $\mathfrak{b}$ be the set of non-oriented bonds of the cubic lattice $\mathfrak{L}$ :

$$
\mathfrak{b}:=\left\{\left\{x, x^{\prime}\right\} \subset \mathfrak{L}:\left|x-x^{\prime}\right|=1\right\} .
$$


Then, $\Omega:=[-1,1]^{\mathfrak{L}} \times \mathbb{D}^{\mathfrak{b}}$ with $\mathbb{D}:=\{z \in \mathbb{C}:|z| \leq 1\}$. I.e., any element of $\Omega$ is a pair $\omega=\left(\omega_{1}, \omega_{2}\right) \in \Omega$, where $\omega_{1}$ is a function of lattice sites with values in $[-1,1]$ and $\omega_{2}$ is a function of bonds with values in the complex closed unit disc $\mathbb{D}$. The first component $\omega_{1}$ is related to the random external static potential and the second component to random hopping amplitudes of fermions. In a subsequent paper we will explicitly fix the probability space $\left(\Omega, \mathfrak{A}_{\Omega}, \mathfrak{a}_{\Omega}\right)$. In the present work, however, all studies are performed for any fixed realization $\omega \in \Omega$ and the specific probability space is unimportant. All results here are uniform w.r.t. the choice of $\omega \in \Omega$.

For any $\omega=\left(\omega_{1}, \omega_{2}\right) \in \Omega, V_{\omega} \in \mathcal{B}\left(\ell^{2}(\mathfrak{L})\right)$ is by definition the self-adjoint multiplication operator with the function $\omega_{1}: \mathfrak{L} \rightarrow[-1,1]$. It represents a bounded static potential. To all $\omega \in \Omega$ and strength $\vartheta \in \mathbb{R}_{0}^{+}$of hopping disorder, we also associate another self-adjoint operator $\Delta_{\omega, \vartheta} \in \mathcal{B}\left(\ell^{2}(\mathfrak{L})\right)$ describing the hoppings of a single particle in the lattice:

$$
\begin{aligned}
{\left[\Delta_{\omega, \vartheta}(\psi)\right](x):=} & 2 d \psi(x)-\sum_{j=1}^{d}\left(\left(1+\vartheta \overline{\omega_{2}\left(\left\{x, x-e_{j}\right\}\right)}\right) \psi\left(x-e_{j}\right)\right. \\
& \left.+\psi\left(x+e_{j}\right)\left(1+\vartheta \omega_{2}\left(\left\{x, x+e_{j}\right\}\right)\right)\right)
\end{aligned}
$$

for any $x \in \mathfrak{L}$ and $\psi \in \ell^{2}(\mathfrak{L})$, with $\left\{e_{k}\right\}_{k=1}^{d}$ being the canonical orthonormal basis of the Euclidian space $\mathbb{R}^{d}$. In the case of vanishing hopping disorder $\vartheta=0$ (up to a minus sign) $\Delta_{\omega, \vartheta}$ is the usual $d$-dimensional discrete Laplacian. Since the hopping amplitudes are complex valued $\left(\omega_{2}\right.$ takes values in $\left.\mathbb{D}\right)$, note additionally that random electromagnetic potentials can be implemented in our model.

Then, for any $\omega \in \Omega$ and parameters $\lambda, \vartheta \in \mathbb{R}_{0}^{+}$, the Hamiltonian describing one quantum particle within a bounded static potential is the discrete Schrödinger operator $\left(\Delta_{\omega, \vartheta}+\lambda V_{\omega}\right)$ acting on the Hilbert space $\ell^{2}(\mathfrak{L})$. The coupling constants $\lambda, \vartheta \in \mathbb{R}_{0}^{+}$represent the strength of disorder of respectively the external static potential and hopping amplitudes.

The time-dependent electromagnetic potential is defined by a compactly supported time-dependent vector potential

$$
\mathbf{A} \in \mathbf{C}_{0}^{\infty}:=\bigcup_{l \in \mathbb{R}^{+}} C_{0}^{\infty}\left(\mathbb{R} \times[-l, l]^{d} ;\left(\mathbb{R}^{d}\right)^{*}\right)
$$

where $\left(\mathbb{R}^{d}\right)^{*}$ is the set of one-forms ${ }^{1}$ on $\mathbb{R}^{d}$ that take values in $\mathbb{R}$. I.e., for some $l \in \mathbb{R}^{+}, \mathbf{A} \in C_{0}^{\infty}\left(\mathbb{R} \times[-l, l]^{d} ;\left(\mathbb{R}^{d}\right)^{*}\right)$ and we use the convention $\mathbf{A}(t, x) \equiv 0$

\footnotetext{
${ }^{1}$ In a strict sense, one should take the dual space of the tangent spaces $T\left(\mathbb{R}^{d}\right)_{x}, x \in \mathbb{R}^{d}$.
} 
whenever $x \notin[-l, l]^{d}$. Since $\mathbf{A} \in \mathbf{C}_{0}^{\infty}, \mathbf{A}(t, x)=0$ for all $t \leq t_{0}$, where $t_{0} \in \mathbb{R}$ is some initial time. The smoothness of $\mathbf{A}$ is not essential in the proofs and is only assumed for simplicity.

We use the Weyl gauge (also named temporal gauge) for the electromagnetic field and as a consequence,

$$
E_{\mathbf{A}}(t, x):=-\partial_{t} \mathbf{A}(t, x), \quad t \in \mathbb{R}, x \in \mathbb{R}^{d},
$$

is the electric field associated with $\mathbf{A}$. We also define the integrated electric field (or electric tension) along the oriented bond $\mathbf{x}:=\left(x^{(1)}, x^{(2)}\right) \in \mathfrak{L}^{2}$ at time $t \in \mathbb{R}$ by

$$
\mathbf{E}_{t}^{\mathbf{A}}(\mathbf{x}):=\int_{0}^{1}\left[E_{\mathbf{A}}\left(t, \alpha x^{(2)}+(1-\alpha) x^{(1)}\right)\right]\left(x^{(2)}-x^{(1)}\right) \mathrm{d} \alpha .
$$

This definition is important because it is the electric field inducing fermionic currents on bonds $\left(x^{(1)}, x^{(2)}\right)$ of nearest neighbors.

To simplify notation, we consider without loss of generality (w.l.o.g.) spinless fermions with negative charge. The cases of particles with spin and positively charged particles can be treated by exactly the same methods. Thus, using the (minimal) coupling of $\mathbf{A} \in \mathbf{C}_{0}^{\infty}$ to the discrete Laplacian, the discrete magnetic Laplacian is (up to a minus sign) the self-adjoint operator

$$
\Delta_{\omega, \vartheta}^{(\mathbf{A})} \equiv \Delta_{\omega, \vartheta}^{(\mathbf{A}(t, \cdot))} \in \mathcal{B}\left(\ell^{2}(\mathfrak{L})\right), \quad t \in \mathbb{R},
$$

defined ${ }^{2}$ by

$$
\left\langle\mathfrak{e}_{x}, \Delta_{\omega, \vartheta}^{(\mathbf{A})} \mathfrak{e}_{y}\right\rangle=\exp \left(i \int_{0}^{1}[\mathbf{A}(t, \alpha y+(1-\alpha) x)](y-x) \mathrm{d} \alpha\right)\left\langle\mathfrak{e}_{x}, \Delta_{\omega, \vartheta} \mathfrak{e}_{y}\right\rangle
$$

for all $t \in \mathbb{R}$ and $x, y \in \mathfrak{L}$. Here, $\langle\cdot, \cdot\rangle$ is the scalar product in $\ell^{2}(\mathfrak{L})$ and $\left\{\mathfrak{e}_{x}\right\}_{x \in \mathfrak{L}}$ is the canonical orthonormal basis $\mathfrak{e}_{x}(y) \equiv \delta_{x, y}$ of $\ell^{2}(\mathfrak{L})$. In (5), similar to (4), $\alpha y+$ $(1-\alpha) x$ and $y-x$ are seen as vectors in $\mathbb{R}^{d}$. In presence of an electromagnetic field associated to an arbitrary vector potential $\mathbf{A} \in \mathbf{C}_{0}^{\infty}$, the one-particle Hamiltonian $\left(\Delta_{\omega, \vartheta}+\lambda V_{\omega}\right)$ at fixed $\omega \in \Omega$ and $\lambda, \vartheta \in \mathbb{R}_{0}^{+}$is replaced with (the time-dependent one)

$$
\Delta_{\omega, \vartheta}^{(\mathbf{A})}+\lambda V_{\omega} \equiv \Delta_{\omega, \vartheta}^{(\mathbf{A}(t, \cdot))}+\lambda V_{\omega}, \quad t \in \mathbb{R}
$$

\footnotetext{
${ }^{2}$ Observe that the sign of the coupling between the electromagnetic potential $\mathbf{A} \in \mathbf{C}_{0}^{\infty}$ and the laplacian is wrong in [BPH1, Eq. (2.8)].
} 


\subsection{CAR $C^{*}$-Algebra of the Infinite Lattice}

Let $\mathcal{P}_{f}(\mathfrak{L}) \subset 2^{\mathfrak{L}}$ be the set of all finite subsets of the $d$-dimensional cubic lattice $\mathfrak{L}$. For any $\Lambda \in \mathcal{P}_{f}(\mathfrak{L}), \mathcal{U}_{\Lambda}$ is the finite dimensional $C^{*}$-algebra generated by 1 and generators $\left\{a_{x, \mathrm{~s}}\right\}_{x \in \Lambda, \mathrm{s} \in \mathrm{S}}$ satisfying the canonical anti-commutation relations, $\mathrm{S}$ being some finite set of spins. As just explained above, the spin dependence of $a_{x, \mathrm{~s}} \equiv a_{x}$ is irrelevant in our proofs (up to trivial modifications) and, w.l.o.g., we only consider spinless fermions, i.e., the case $\mathrm{S}=\{0\}$.

Let

$$
\Lambda_{L}:=\left\{\left(x_{1}, \ldots, x_{d}\right) \in \mathfrak{L}:\left|x_{1}\right|, \ldots,\left|x_{d}\right| \leq L\right\} \in \mathcal{P}_{f}(\mathfrak{L})
$$

for all $L \in \mathbb{R}^{+}$and observe that $\left\{\mathcal{U}_{\Lambda_{L}}\right\}_{L \in \mathbb{R}^{+}}$is an increasing net of $C^{*}$-algebras. Hence, the set

$$
\mathcal{U}_{0}:=\bigcup_{L \in \mathbb{R}^{+}} \mathcal{U}_{\Lambda_{L}}
$$

of local elements is a normed $*$-algebra with $\|A\|_{\mathcal{U}_{0}}=\|A\|_{\mathcal{U}_{\Lambda_{L}}}$ for all $A \in \mathcal{U}_{\Lambda_{L}}$ and $L \in \mathbb{R}^{+}$. The CAR $C^{*}$-algebra $\mathcal{U}$ of the infinite system with norm $\|\cdot\|_{\mathcal{U}}$ is by definition the completion of the normed $*$-algebra $\mathcal{U}_{0}$. It is separable, by finite dimensionality of $\mathcal{U}_{\Lambda}$ for $\Lambda \in \mathcal{P}_{f}(\mathfrak{L})$.

For any fixed $\theta \in \mathbb{R} /(2 \pi \mathbb{Z})$, the condition

$$
\sigma_{\theta}\left(a_{x}\right)=\mathrm{e}^{-i \theta} a_{x}
$$

defines a unique automorphism $\sigma_{\theta}$ of the $C^{*}$-algebra $\mathcal{U}$. A special role is played by $\sigma_{\pi}$. Elements $B_{1}, B_{2} \in \mathcal{U}$ satisfying $\sigma_{\pi}\left(B_{1}\right)=B_{1}$ and $\sigma_{\pi}\left(B_{2}\right)=-B_{2}$ are respectively called even and $o d d$, while elements $B \in \mathcal{U}$ satisfying $\sigma_{\theta}(B)=B$ for any $\theta \in[0,2 \pi)$ are called gauge invariant. The set

$$
\mathcal{U}^{+}:=\left\{B \in \mathcal{U}: B=\sigma_{\pi}(B)\right\} \subset \mathcal{U}
$$

of all even elements is a $*$-algebra. By continuity of $\sigma_{\theta}$, it follows that $\mathcal{U}^{+}$is closed and hence a $C^{*}$-algebra.

\subsection{Banach Space of Short-Range Interactions}

An interaction is a family $\Phi=\left\{\Phi_{\Lambda}\right\}_{\Lambda \in \mathcal{P}_{f}(\mathfrak{L})}$ of even and self-adjoint local elements $\Phi_{\Lambda}=\Phi_{\Lambda}^{*} \in \mathcal{U}^{+} \cap \mathcal{U}_{\Lambda}$ with $\Phi_{\emptyset}=0$. Obviously, the set of all interactions can be endowed with a real vector space structure:

$$
\left(\alpha_{1} \Phi+\alpha_{2} \Psi\right)_{\Lambda}:=\alpha_{1} \Phi_{\Lambda}+\alpha_{2} \Psi_{\Lambda}, \quad \Lambda \in \mathcal{P}_{f}(\mathfrak{L})
$$


for any interactions $\Phi, \Psi$, and any real numbers $\alpha_{1}, \alpha_{2}$. We define Banach spaces of short-range interactions by introducing specific norms for interactions, taking into account space decay.

To this end, as done in [BP3] by following [NOS, Eqs. (1.3)-(1.4)], we consider positive-valued and non-increasing decay functions $\mathbf{F}: \mathbb{R}_{0}^{+} \rightarrow \mathbb{R}^{+}$satisfying the following properties:

- Summability on $\mathfrak{L}$.

$$
\|\mathbf{F}\|_{1, \mathfrak{L}}:=\sup _{y \in \mathfrak{L}} \sum_{x \in \mathfrak{L}} \mathbf{F}(|x-y|)=\sum_{x \in \mathfrak{L}} \mathbf{F}(|x|)<\infty .
$$

- Bounded convolution constant.

$$
\mathbf{D}:=\sup _{x, y \in \mathfrak{L}} \sum_{z \in \mathfrak{L}} \frac{\mathbf{F}(|x-z|) \mathbf{F}(|z-y|)}{\mathbf{F}(|x-y|)}<\infty .
$$

A typical example of such a $\mathbf{F}$ for $\mathfrak{L}=\mathbb{Z}^{d}, d \in \mathbb{N}$, is the function

$$
\mathbf{F}(r)=(1+r)^{-(d+\epsilon)}, \quad r \in \mathbb{R}_{0}^{+},
$$

which has convolution constant $\mathbf{D} \leq 2^{d+1+\epsilon}\|\mathbf{F}\|_{1, \mathfrak{L}}$ for $\epsilon \in \mathbb{R}^{+}$. See [NOS, Eq. (1.6)] or [Si, Example 3.1]. Note that the exponential function $\mathbf{F}(r)=\mathrm{e}^{-\varsigma r}, \varsigma \in$ $\mathbb{R}^{+}$, satisfies (10) but not (11). Nevertheless, for every function $\mathbf{F}$ with bounded convolution constant (11) and any strictly positive parameter $\varsigma \in \mathbb{R}^{+}$, the function

$$
\tilde{\mathbf{F}}(r)=\mathrm{e}^{-\varsigma r} \mathbf{F}(r), \quad r \in \mathbb{R}_{0}^{+},
$$

clearly satisfies Condition (11) with a convolution constant that is no bigger than the one of F. In fact, as observed in [Si, Section 3.1], the multiplication of such a function $\mathbf{F}$ with a non-increasing weight $f: \mathbb{R}_{0}^{+} \rightarrow \mathbb{R}^{+}$satisfying $f(r+s) \geq$ $f(r) f(s)$ (logarithmically superadditive function) produces a new positive-valued and non-increasing decay function without increasing the convolution constant $\mathbf{D}$. In all the paper, (10)-(11) are assumed to be satisfied.

The function $\mathbf{F}$ encodes the short-range property of interactions. Indeed, an interaction $\Phi$ is said to be short-range if

$$
\|\Phi\|_{\mathcal{W}}:=\sup _{x, y \in \mathfrak{L}} \sum_{\Lambda \in \mathcal{P}_{f}(\mathfrak{L}), \Lambda \supset\{x, y\}} \frac{\left\|\Phi_{\Lambda}\right\|_{\mathcal{U}}}{\mathbf{F}(|x-y|)}<\infty .
$$


The map $\Phi \mapsto\|\Phi\|_{\mathcal{W}}$ defines a norm on interactions and the space of short-range interactions w.r.t. to the decay function $\mathbf{F}$ is, by definition, the real separable Banach space $\mathcal{W} \equiv\left(\mathcal{W},\|\cdot\|_{\mathcal{W}}\right)$ of all interactions $\Phi$ with $\|\Phi\|_{\mathcal{W}}<\infty$. It turns out that all $\Phi \in \mathcal{W}$ define, in a natural way, infinite-volume quantum dynamics, i.e., they define $C^{*}$-dynamical systems on $\mathcal{U}$. This fact is discussed in detail in [BP3, Section 3.1].

\subsection{Unperturbed Dynamics of Interacting Fermions}

To any $\omega \in \Omega$, hopping and potential strengths $\vartheta \in \mathbb{R}_{0}^{+}$, we associate a shortrange interaction $\Psi^{(\omega, \vartheta)} \in \mathcal{W}$ defined as follows: Fix $\Psi^{\mathrm{IP}} \in \mathcal{W}$. Then,

$$
\Psi_{\Lambda}^{(\omega, \vartheta)}:=\left\langle\mathfrak{e}_{x}, \Delta_{\omega, \vartheta} \mathfrak{e}_{y}\right\rangle a_{x}^{*} a_{y}+\left(1-\delta_{x, y}\right)\left\langle\mathfrak{e}_{y}, \Delta_{\omega, \vartheta} \mathfrak{e}_{x}\right\rangle a_{y}^{*} a_{x}+\Psi_{\{x, y\}}^{\mathrm{IP}} \in \mathcal{U}^{+} \cap \mathcal{U}_{\Lambda}
$$

whenever $\Lambda=\{x, y\}$ for $x, y \in \mathfrak{L}$, and $\Psi_{\Lambda}^{(\omega, \vartheta)}:=\Psi_{\Lambda}^{\mathrm{IP}}$ otherwise. Hence, in presence of bounded static potentials, the internal energy observable $H_{L}^{(\omega, \vartheta, \lambda)} \in$ $\mathcal{U}^{+} \cap \mathcal{U}_{\Lambda}$ in the box $\Lambda_{L}$ of the interacting inhomogeneous fermion system is defined by

$$
\begin{aligned}
H_{L}^{(\omega, \vartheta, \lambda)} & :=\sum_{\Lambda \subset \Lambda_{L}} \Psi_{\Lambda}^{(\omega, \vartheta)}+\lambda \sum_{x \in \Lambda_{L}} \omega_{1}(x) a_{x}^{*} a_{x} \\
& =\sum_{x, y \in \Lambda_{L}}\left\langle\mathfrak{e}_{x},\left(\Delta_{\omega, \vartheta}+\lambda V_{\omega}\right) \mathfrak{e}_{y}\right\rangle a_{x}^{*} a_{y}+\sum_{\Lambda \subset \Lambda_{L}} \Psi_{\Lambda}^{\mathrm{IP}}
\end{aligned}
$$

for $\omega=\left(\omega_{1}, \omega_{2}\right) \in \Omega, \vartheta, \lambda \in \mathbb{R}_{0}^{+}$and $L \in \mathbb{R}^{+}$. Observe that the first sum in the right hand side (r.h.s.) of the second equality in (14) is the second quantization of the one-particle operator $\Delta_{\omega, \vartheta}+\lambda V_{\omega}$ restricted to the subspace $\ell^{2}\left(\Lambda_{L}\right) \subset \ell^{2}(\mathfrak{L})$. The second sum in that equality encodes all interaction mechanisms involving more than one particle, in the box $\Lambda_{L}$.

The finite volume dynamics thus corresponds to the continuous group $\left\{\tau_{t}^{(L)}\right\}_{t \in \mathbb{R}}$ of $*$-automorphisms defined by

$$
\tau_{t}^{(\omega, \vartheta, \lambda, L)}(B)=\mathrm{e}^{i t H_{L}^{(\omega, \vartheta, \lambda)}} B \mathrm{e}^{-i t H_{L}^{(\omega, \vartheta, \lambda)}}, \quad B \in \mathcal{U},
$$

for any $t \in \mathbb{R}, \omega=\left(\omega_{1}, \omega_{2}\right) \in \Omega, \vartheta, \lambda \in \mathbb{R}_{0}^{+}$and $L \in \mathbb{R}^{+}$.

As explained in [NS] for quantum spin systems via Lieb-Robinson bounds, it is clear that the strong limit $L \rightarrow \infty$ of $\tau_{t}^{(\omega, \vartheta, \lambda, L)}$ is well-defined. Here, we apply [BP3, Theorem 3.6], which yields the following assertions: 


\section{Theorem 2.1 (Infinite volume dynamics and its generator)}

Assume (10)-(11). Let $\omega \in \Omega$ and $\vartheta_{0}, \vartheta, \lambda \in \mathbb{R}_{0}^{+}$.

(i) Infinite volume dynamics. The continuous groups $\left\{\tau_{t}^{(\omega, \vartheta, \lambda, L)}\right\}_{t \in \mathbb{R}}, L \in \mathbb{R}^{+}$, converge strongly to a $C_{0}$-group $\left\{\tau_{t}^{(\omega, \vartheta, \lambda)}\right\}_{t \in \mathbb{R}}$ of $*$-automorphisms with generator $\delta^{(\omega, \vartheta, \lambda)}$, as $L \rightarrow \infty$.

(ii) Infinitesimal generator. $\delta^{(\omega, \vartheta, \lambda)}$ is a conservative closed symmetric derivation which is equal on its core $\mathcal{U}_{0}$ to

$\delta^{(\omega, \vartheta, \lambda)}(B)=i \sum_{x, y \in \mathfrak{L}}\left\langle\mathfrak{e}_{x},\left(\Delta_{\omega, \vartheta}+\lambda V_{\omega}\right) \mathfrak{e}_{y}\right\rangle\left[a_{x}^{*} a_{y}, B\right]+i \sum_{\Lambda \in \mathcal{P}_{f}(\mathfrak{L})}\left[\Psi_{\Lambda}^{\mathrm{IP}}, B\right], \quad B \in \mathcal{U}_{0}$

Both infinite sums in the above equation converge absolutely.

(iii) Lieb-Robinson bounds. For any $\vartheta \in\left[0, \vartheta_{0}\right], t \in \mathbb{R}, B_{1} \in \mathcal{U}^{+} \cap \mathcal{U}_{\Lambda^{(1)}}$ and $B_{2} \in \mathcal{U}_{\Lambda^{(2)}}$ with disjoint sets $\Lambda^{(1)}, \Lambda^{(2)} \in \mathcal{P}_{f}(\mathfrak{L})$,

$$
\begin{aligned}
\left\|\left[\tau_{t}^{(\omega, \vartheta, \lambda)}\left(B_{1}\right), B_{2}\right]\right\|_{\mathcal{U}} \leq & 2 \mathbf{D}^{-1}\left\|B_{1}\right\|_{\mathcal{U}}\left\|B_{2}\right\|_{\mathcal{U}}\left(\mathrm{e}^{2 \mathbf{D}|t| D_{\vartheta_{0}}}-1\right) \\
& \times \sum_{x \in \Lambda^{(1)}} \sum_{y \in \Lambda^{(2)}} \mathbf{F}(|x-y|)
\end{aligned}
$$

where

$$
D_{\vartheta_{0}}:=\sup \left\{\left\|\Psi^{(\omega, \vartheta)}\right\|_{\mathcal{W}}: \omega \in \Omega, \vartheta \in\left[0, \vartheta_{0}\right]\right\}<\infty
$$

If $\Psi^{\mathrm{IP}}, \vartheta=0$ in $\Psi^{(\omega, \vartheta)}$ then $\tau^{(\omega, \vartheta, \lambda)}$ becomes a family of Bogoliubov automorphisms of $\mathcal{U}$, as described in [BPH1, BPH2, BPH3, BPH4] for homogeneous hopping terms. Meanwhile, density-density interactions resulting from the second quantization of two-body interactions, like for instance

$$
\sum_{x, y} v(|x-y|) a_{y}^{*} a_{y} a_{x}^{*} a_{x}
$$

where $v(r): \mathbb{R}_{0}^{+} \rightarrow \mathbb{R}^{+}$is a real-valued function such that

$$
\sup _{r \in \mathbb{R}_{0}^{+}}\left\{\frac{v(r)}{\mathbf{F}(r)}\right\}<\infty,
$$

can be handled in our setting. Hence, (considering fermions with spins $\uparrow$ or $\downarrow$ ) our setting includes the celebrated Hubbard model. The Coulomb potential is 
excluded from our analysis because it is not summable, see Condition (10). The function

$$
v(r)=D \frac{\mathrm{e}^{-m r}}{1+r}, \quad r \in \mathbb{R}_{0}^{+}, D, m \in \mathbb{R}^{+},
$$

which is similar to the Yukawa potential for some mass $m \in \mathbb{R}^{+}$, is allowed by taking, for instance, the function $\mathbf{F}(r)=D \mathrm{e}^{-\varsigma r}(1+r)^{-(d+1)}$ with $\varsigma \in(0, m)$.

The potential (17) is the physical example we have in mind. Indeed, it is believed in theoretical physics that the Coulomb potential is screened by the positively charged ions which form the lattice $\mathfrak{L}$. In [GV, Section 1.3.2] the authors assert that one should first consider models with Yukawa potentials (17) (or even finite range) to perform the thermodynamic limit and next the limit $m \downarrow 0$ to recover the physical model. This procedure is only justified a posteriori and we do not consider here the highly non-trivial mathematical problem of Coulomb interactions within a mixture of electrons and ions. Note additionally that, as we are using the lattice $\mathfrak{L}:=\mathbb{Z}^{d}$ to represent space, (17) does not have a singularity at $r=0$, in contrast with the Coulomb and Yukawa potentials in the continuous space $\mathbb{R}^{d}$.

\subsection{Dynamics Driven by Time-Dependent Electromagnetic Fields}

When the electromagnetic field is switched on, i.e., for $t \geq t_{0}$, the total energy observable in a box $\Lambda_{L}$ that includes the region where the electromagnetic field does not vanish equals

$$
H_{L}^{(\omega, \vartheta, \lambda)}+W_{t}^{(\omega, \vartheta, \mathbf{A})},
$$

where, for any $\omega \in \Omega, \vartheta \in \mathbb{R}_{0}^{+}, \mathbf{A} \in \mathbf{C}_{0}^{\infty}$ and $t \in \mathbb{R}$,

$$
W_{t}^{(\omega, \vartheta, \mathbf{A})}:=\sum_{x, y \in \mathfrak{L}}\left\langle\mathfrak{e}_{x},\left(\Delta_{\omega, \vartheta}^{(\mathbf{A})}-\Delta_{\omega, \vartheta}\right) \mathfrak{e}_{y}\right\rangle a_{x}^{*} a_{y} \in \mathcal{U}^{+} \cap \mathcal{U}_{0}
$$

is the electromagnetic potential energy observable. The finite volume dynamics becomes non-autonomous in presence of electromagnetic fields.

Indeed, for any time $t \in \mathbb{R}$, consider the conservative closed symmetric derivation

$$
\delta_{t}^{(\omega, \vartheta, \lambda, \mathbf{A})}:=\delta^{(\omega, \vartheta, \lambda)}+i\left[W_{t}^{(\omega, \vartheta, \mathbf{A})}, \cdot\right],
$$

where we recall that $\delta^{(\omega, \vartheta, \lambda)}$ is the generator of the one-parameter group $\tau^{(\omega, \vartheta, \lambda)}:=$ $\left\{\tau_{t}^{(\omega, \vartheta, \lambda)}\right\}_{t \in \mathbb{R}}$ of $*$-automorphisms, see Theorem 2.1. Define also the family

$$
\left\{\mathfrak{U}_{t, s}\right\}_{s, t \in \mathbb{R}} \subset \operatorname{Dom}\left(\delta^{(\omega, \vartheta, \lambda)}\right)
$$


of unitary elements by the absolutely summable series, for any $s, t \in \mathbb{R}$,

$\mathfrak{U}_{t, s}:=\mathbf{1}+\sum_{k \in \mathbb{N}}(-i)^{k} \int_{s}^{t} \mathrm{~d} s_{1} \cdots \int_{s}^{s_{k-1}} \mathrm{~d} s_{k} \tau_{s_{1}-t}^{(\omega, \vartheta, \lambda)}\left(W_{s_{1}}^{(\omega, \vartheta, \mathbf{A})}\right) \cdots \tau_{s_{k}-t}^{(\omega, \vartheta, \lambda)}\left(W_{s_{k}}^{(\omega, \vartheta, \mathbf{A})}\right)$.

As explained in [BPH1, Eqs. (5.18)-(5.20)], this series absolutely converges in the Banach spaces $\mathcal{U}$ and

$$
\left(\operatorname{Dom}\left(\delta^{(\omega, \vartheta, \lambda)}\right),\|\cdot\|_{\delta^{(\omega, \vartheta, \lambda)}}\right)
$$

$\|\cdot\|_{\delta(\omega, \vartheta, \lambda)}$ being the graph norm of the closed operator $\delta^{(\omega, \vartheta, \lambda)}$ with domain $\operatorname{Dom}\left(\delta^{(\omega, \vartheta, \lambda)}\right)$.

Now, since $\mathbf{A} \in \mathbf{C}_{0}^{\infty}$, the map $t \mapsto W_{t}^{(\omega, \vartheta, \mathbf{A})}$ from $\mathbb{R}$ to $\mathcal{U}_{0}$ is smooth and [BP3, Corollary 4.2] ensures the existence of the infinite volume non-autonomous dynamics:

\section{Theorem 2.2 (Non-autonomous dynamics)}

Assume (10)-(11). Let $\omega \in \Omega, \vartheta, \lambda \in \mathbb{R}_{0}^{+}$and $\mathbf{A} \in \mathbf{C}_{0}^{\infty}$. Then, there is a strongly continuous two-parameter family $\left\{\tau_{t, s}^{(\omega, \vartheta, \lambda, \mathbf{A})}\right\}_{s, t \in \mathbb{R}}$ of $*$-automorphisms on $\mathcal{U}$ satisfying the following properties:

(i) Reverse "cocycle" property.

$$
\forall s, r, t \in \mathbb{R}: \quad \tau_{t, s}^{(\omega, \vartheta, \lambda, \mathbf{A})}=\tau_{r, s}^{(\omega, \vartheta, \lambda, \mathbf{A})} \tau_{t, r}^{(\omega, \vartheta, \lambda, \mathbf{A})} .
$$

(ii) Non-autonomous evolution equation. It is the unique strongly continuous two-parameter family of bounded operators on $\mathcal{U}$ satisfying, in the strong sense on the dense domain $\mathcal{U}_{0} \subset \mathcal{U}$,

$$
\forall s, t \in \mathbb{R}: \quad \partial_{t} \tau_{t, s}^{(\omega, \vartheta, \lambda, \mathbf{A})}=\tau_{t, s}^{(\omega, \vartheta, \lambda, \mathbf{A})} \circ \delta_{t}^{(\omega, \vartheta, \lambda, \mathbf{A})}, \quad \tau_{s, s}^{(\omega, \vartheta, \lambda, \mathbf{A})}=\mathbf{1}_{\mathcal{U}} .
$$

(iii) Interaction picture. For any $s, t \in \mathbb{R}$,

$$
\tau_{t, s}^{(\omega, \vartheta, \lambda, \mathbf{A})}(B)=\tau_{t-s}^{(\omega, \vartheta, \lambda)}\left(\mathfrak{U}_{t, s}^{*} B \mathfrak{U}_{t, s}\right), \quad B \in \mathcal{U}
$$

Proof: (i)-(ii) is a direct application of [BP3, Corollary 4.2]. We omit the details. Moreover, for any $\mathbf{A} \in \mathbf{C}_{0}^{\infty}$, the coefficients

$$
\mathbf{w}_{x, y}(\eta, t):=\left\langle\mathfrak{e}_{x}, \vartheta\left(\Delta_{\omega}^{(\eta \mathbf{A})}-\Delta_{\omega}\right) \mathfrak{e}_{y}\right\rangle, \quad x, y \in \mathfrak{L},
$$


of the electromagnetic potential energy observable $W_{t}^{(\omega, \vartheta, \eta \mathbf{A})}$ are complex-valued functions of $(\eta, t) \in \mathbb{R}^{2}$ that satisfy

$$
\overline{\mathbf{w}_{x, y}(\eta, t)}=\mathbf{w}_{y, x}(\eta, t) \quad \text { and } \quad \mathbf{w}_{x, x+z}(0, t)=0
$$

for all $x, y, z \in \mathfrak{L}$ and $(\eta, t) \in \mathbb{R}^{2}$. By the mean value theorem, $\left\{\mathbf{w}_{x, y}\right\}_{x, y \in \mathfrak{L}}$ are also uniformly bounded and Lipschitz continuous (as functions of times): There is a constant $D \in \mathbb{R}^{+}$such that, for all $\vartheta_{0} \in \mathbb{R}^{+}, \omega \in \Omega, \vartheta \in\left[0, \vartheta_{0}\right], \eta \in \mathbb{R}$, and $s, t \in \mathbb{R}$,

$$
\sup _{x, y \in \mathfrak{L}}\left|\mathbf{w}_{x, y}(\eta, t)-\mathbf{w}_{x, y}(\eta, s)\right| \leq D \eta|t-s|, \quad \sup _{x, y \in \mathfrak{L}} \sup _{\eta, t \in \mathbb{R}}\left|\mathbf{w}_{x, y}(\eta, t)\right| \leq D .
$$

From (20) and [BP3, Eq. (145), Theorem 4.7 (ii)], we arrive at Assertion (iii).

Again, for $\Psi^{\mathrm{IP}}, \vartheta=0,\left\{\tau_{t, s}^{(\omega, \vartheta, \lambda, \mathbf{A})}\right\}_{t \geq s}$ is the two-parameter family of Bogoliubov automorphisms of $\mathcal{U}$ described in [BPH1, BPH2, BPH3, BPH4] for homogeneous hopping terms.

\subsection{Time-Dependent State of the System}

Thermal equilibrium states are defined here to be completely passive states. This definition [PW, Definitions 1.1, 1.3] is based on the 2nd law of thermodynamics. In [BP4], the complete passivity of states will be discussed with much more details. By [PW, Theorem 1.4], such states are $\left(\tau^{(\omega, \vartheta, \lambda)}, \beta\right)-\mathrm{KMS}$ states for some inverse temperature, or time scale (cf. [BP4]), $\beta \in[0, \infty]$.

The case $\beta=0$ corresponds to the $\tau^{(\omega, \vartheta, \lambda)}$-invariant traces, also called chaotic states, whereas the limiting case $\beta=\infty$ refers to ground states. For simplicity, we exclude both extreme cases and only consider fermion systems at finite inverse temperature $\beta \in \mathbb{R}^{+}$. Note also that, in some situations, the parameter $\beta$ may not be arbitrarily chosen, as illustrated in [BR2, Example 5.3.27.]. In fact, it is not even a priori clear that thermal equilibrium states, in the above sense, exist for arbitrary interacting fermion systems. As already mentioned above, the dynamics $\tau^{(\omega, \vartheta, \lambda)}$ is such that, for all $\beta \in \mathbb{R}^{+}$, there is at least one $\left(\tau^{(\omega, \vartheta, \lambda)}, \beta\right)$-KMS state.

Indeed, by Theorem 2.1 (i), the continuous group $\left\{\tau_{t}^{(\omega, \vartheta, \lambda, L)}\right\}_{t \in \mathbb{R}}$ of $*$-automorphisms of $\mathcal{U}$ defined by (15) converges strongly to the $C_{0}$-group $\tau^{(\omega, \vartheta, \lambda)}:=$ $\left\{\tau_{t}^{(\omega, \vartheta, \lambda)}\right\}_{t \in \mathbb{R}}$. Moreover, it is well-known that, at finite volume and any inverse temperature $\beta \in \mathbb{R}^{+}$, the corresponding Gibbs state is the unique KMS state associated with $\left\{\tau_{t}^{(\omega, \vartheta, \lambda, L)}\right\}_{t \in \mathbb{R}}$. Hence, by [BR2, Proposition 5.3.25.], there is a 
$\left(\tau^{(\omega, \vartheta, \lambda)}, \beta\right)$-KMS state $\varrho^{(\beta, \omega, \vartheta, \lambda)}$ for every $\beta \in \mathbb{R}^{+}$. Depending on the interaction $\Psi^{\mathrm{IP}}$ in $\Psi^{(\omega, \vartheta)}$, the sequence of Gibbs states can have a priori several weak*accumulation points and all these limit states are $\left(\tau^{(\omega, \vartheta, \lambda)}, \beta\right)-\mathrm{KMS}$. As a consequence, the so-called KMS condition (and thus the completely passivity of states) does not uniquely define the thermal equilibrium state of the system in infinite volume.

It is easy to check that the set $\mathfrak{Q}^{(\beta, \omega, \vartheta, \lambda)} \subset \mathcal{U}^{*}$ of $\left(\tau^{(\omega, \vartheta, \lambda)}, \beta\right)-\mathrm{KMS}$ states is a non-empty, sequentially weak ${ }^{*}$-compact and convex set. For any $\beta \in \mathbb{R}^{+}, \omega \in \Omega$ and $\vartheta, \lambda \in \mathbb{R}_{0}^{+}, \varrho^{(\beta, \omega, \vartheta, \lambda)}$ is, by definition, an arbitrary element of $\mathfrak{Q}^{(\beta, \omega, \vartheta, \lambda)}$ fixed once and for all. It represents a thermal equilibrium state of the system before the electromagnetic field is switched on.

Since $\mathbf{A}(t, x)=0$ for all $t \leq t_{0}$, similar to [BPH1, BPH2, BPH3, BPH4], the time evolution of the state of the system thus equals

$$
\rho_{t}^{(\beta, \omega, \vartheta, \lambda, \mathbf{A})}:=\left\{\begin{array}{lll}
\varrho^{(\beta, \omega, \vartheta, \lambda)} & , & t \leq t_{0}, \\
\varrho^{(\beta, \omega, \vartheta, \lambda)} \circ \tau_{t, t_{0}}^{(\omega, \vartheta, \lambda, \mathbf{A})} & , & t \geq t_{0},
\end{array}\right.
$$

for any $\beta \in \mathbb{R}^{+}, \omega \in \Omega, \vartheta, \lambda \in \mathbb{R}_{0}^{+}$and $\mathbf{A} \in \mathbf{C}_{0}^{\infty}$. By stationarity of KMS states, the definition does not depend on the particular choice of initial time $t_{0}$ with $\mathbf{A}(t, x)=0$ for all $t \leq t_{0}$. This time-dependent state is generally not quasifree unless $\Psi^{\mathrm{IP}}=0$ in $\Psi^{(\omega, \vartheta)}$.

\section{Heat Production and Current Linear Response}

In this section we extend all results of [BPH1, BPH2] to fermion systems with short-range interactions. This is possible because of Lieb-Robinson bounds for multi-commutators, which yield [BP3, Theorems 4.8-4.9]. By using these results, we can follow the arguments of [BPH1, BPH2] to prove, exactly in the same way, all their assertions in the interacting case. Therefore, we refrain from giving all the detailed proofs and we shorten our discussions by referring to [BPH1, BPH2] for details.

\subsection{Heat Production}

Similar to [BPH1] we analyze the effect of electromagnetic fields in terms of heat production within the fermion system. This study is related to Joule's law, which describes the rate at which resistance converts electric energy into heat. Its 
mathematical formulation requires Araki's notion of relative entropy [A1, A2]. See [BPH1, Section A.1] for a concise account on the relative entropy in $C^{*}$ algebras.

In the case of the $C^{*}$-algebra $\mathcal{U}$, the quantum relative entropy takes a simple form by using the net of finite dimensional $C^{*}$-algebras $\left\{\mathcal{U}_{\Lambda}\right\}_{\Lambda \in \mathcal{P}_{f}(\mathfrak{L})}$ generating $\mathcal{U}$. Let $\Lambda \in \mathcal{P}_{f}(\mathfrak{L})$ and denote by tr the normalized trace on $\mathcal{U}_{\Lambda}$, also named the tracial state of $\mathcal{U}_{\Lambda}$. For any state $\rho_{\Lambda} \in \mathcal{U}_{\Lambda}^{*}$, there is a unique adjusted density matrix $\mathrm{d}_{\rho_{\Lambda}} \in \mathcal{U}$, that is, $\mathrm{d}_{\rho_{\Lambda}} \geq 0, \operatorname{tr}\left(\mathrm{d}_{\rho_{\Lambda}}\right)=1$ and $\rho_{\Lambda}(A)=\operatorname{tr}\left(\mathrm{d}_{\rho_{\Lambda}} A\right)$ for all $A \in \mathcal{U}_{\Lambda}$. See, for instance, [AM, Lemma 3.1 (i)]. We define by $\operatorname{supp}\left(\rho_{\Lambda}\right)$ the smallest projection $\mathrm{P} \in \mathcal{U}_{\Lambda}$ such that $\rho_{\Lambda}(\mathrm{P})=1$. Then, the relative entropy of a state $\rho_{1, \Lambda} \in \mathcal{U}_{\Lambda}^{*}$ w.r.t. $\rho_{2, \Lambda} \in \mathcal{U}_{\Lambda}^{*}$ is defined by

$S_{\mathcal{U}_{\Lambda}}\left(\rho_{1, \Lambda} \mid \rho_{2, \Lambda}\right):= \begin{cases}\rho_{1, \Lambda}\left(\ln \mathrm{d}_{\rho_{1, \Lambda}}-\ln \mathrm{d}_{\rho_{2, \Lambda}}\right) & , \text { if } \operatorname{supp}\left(\rho_{2, \Lambda}\right) \geq \operatorname{supp}\left(\rho_{1, \Lambda}\right), \\ +\infty & \text { otherwise }\end{cases}$

under the convention $\left.x \ln x\right|_{x=0}:=0$. We then define the relative entropy of any state $\rho_{1} \in \mathcal{U}^{*}$ w.r.t. $\rho_{2} \in \mathcal{U}^{*}$ by

$$
\mathrm{S}\left(\rho_{1} \mid \rho_{2}\right):=\lim _{L \rightarrow \infty} S_{\mathcal{U}_{\Lambda_{L}}}\left(\rho_{1, \Lambda_{L}} \mid \rho_{2, \Lambda_{L}}\right)=\sup _{L \in \mathbb{R}^{+}} S_{\mathcal{U}_{\Lambda_{L}}}\left(\rho_{1, \Lambda_{L}} \mid \rho_{2, \Lambda_{L}}\right) \in[0, \infty]
$$

with $\rho_{1, \Lambda_{L}}$ and $\rho_{2, \Lambda_{L}}$ being the restrictions to $\mathcal{U}_{\Lambda_{L}}$ of the states $\rho_{1}$ and $\rho_{2}$, respectively. As discussed in [BPH1], this limit exists and equals Araki's relative entropy. In particular, it is a non-negative (possibly infinite) quantity. With this, the heat production is defined as follows:

\section{Definition 3.1 (Heat production)}

For any $\beta \in \mathbb{R}^{+}, \omega \in \Omega, \vartheta, \lambda \in \mathbb{R}_{0}^{+}$and $\mathbf{A} \in \mathbf{C}_{0}^{\infty}, \mathbf{Q}^{(\omega, \mathbf{A})} \equiv \mathbf{Q}^{(\beta, \omega, \vartheta, \lambda, \mathbf{A})}$ is defined as a map from $\mathbb{R}$ to $\overline{\mathbb{R}}$ by

$$
\mathbf{Q}^{(\omega, \mathbf{A})}(t):=\beta^{-1} \mathrm{~S}\left(\rho_{t}^{(\beta, \omega, \vartheta, \lambda, \mathbf{A})} \mid \varrho^{(\beta, \omega, \vartheta, \lambda)}\right) \in[0, \infty] .
$$

In [BPH1, Theorem 3.2], that is, for $\Psi^{\mathrm{IP}}, \vartheta=0$ in the definition of $\Psi^{(\omega, \vartheta)}$, we prove the 1st law of thermodynamics for the system under consideration, implying that the heat production generated by electromagnetic fields is exactly the increase of the internal energy resulting from the modification of the (infinite volume) state of the system. Such a result is generalized for the setting considered here. Indeed, the arguments of [BPH1, Sections 5.3-5.4] - which proves [BPH1, Theorem 3.2] - still work, provided interparticle interactions have some sufficiently fast polynomial decay. More precisely, this means the following condition on the positive and non-increasing function $\mathbf{F}: \mathbb{R}_{0}^{+} \rightarrow \mathbb{R}^{+}$: 
- Polynomial decay. There is a constant $\varsigma>2 d$ and, for all $m \in \mathbb{N}_{0}$, an absolutely summable sequence $\left\{\mathbf{u}_{n, m}\right\}_{n \in \mathbb{N}} \in \ell^{1}(\mathbb{N})$ such that, for all $n \in \mathbb{N}$ with $n>m$,

$$
\left|\Lambda_{n} \backslash \Lambda_{n-1}\right| \sum_{z \in \Lambda_{m}} \max _{y \in \Lambda_{n} \backslash \Lambda_{n-1}} \mathbf{F}(|z-y|) \leq \frac{\mathbf{u}_{n, m}}{(1+n)^{\varsigma}} .
$$

Examples of functions $\mathbf{F}: \mathbb{R}_{0}^{+} \rightarrow \mathbb{R}^{+}$satisfying (10)-(11) and (24) are obviously given by (12) for sufficiently large parameters $\epsilon \in \mathbb{R}^{+}$. Observe that Condition (24) is assumed in [BP3, Theorem 4.8].

To present now the result, in particular the derivation of the 1st law of thermodynamics, similar to [BPH1, Section 3.2] we need to define the total, internal, and electromagnetic potential energies: For any $\beta \in \mathbb{R}^{+}, \omega \in \Omega, \vartheta, \lambda \in \mathbb{R}_{0}^{+}$, $\mathbf{A} \in \mathbf{C}_{0}^{\infty}$ and $t \in \mathbb{R}$, the total energy increment due to the interaction of the charged fermions with the electromagnetic field equals

$$
\begin{aligned}
& \lim _{L \rightarrow \infty}\left\{\rho_{t}^{(\beta, \omega, \vartheta, \lambda, \mathbf{A})}\left(H_{L}^{(\omega, \vartheta, \lambda)}+W_{t}^{(\omega, \vartheta, \mathbf{A})}\right)-\varrho^{(\beta, \omega, \vartheta, \lambda)}\left(H_{L}^{(\omega, \vartheta, \lambda)}\right)\right\} \\
= & \mathbf{S}^{(\omega, \mathbf{A})}(t)+\mathbf{P}^{(\omega, \mathbf{A})}(t),
\end{aligned}
$$

where $H_{L}^{(\omega, \vartheta, \lambda)}$ and $W_{t}^{(\omega, \vartheta, \mathbf{A})}$ are respectively the internal energy observable (14) and the electromagnetic potential energy observable (18). Here, $\mathbf{S}^{(\omega, \mathbf{A})} \equiv \mathbf{S}^{(\beta, \omega, \vartheta, \lambda, \mathbf{A})}$ is the internal energy increment defined by

$$
\mathbf{S}^{(\omega, \mathbf{A})}(t):=\lim _{L \rightarrow \infty}\left\{\rho_{t}^{(\beta, \omega, \vartheta, \lambda, \mathbf{A})}\left(H_{L}^{(\omega, \vartheta, \lambda)}\right)-\varrho^{(\beta, \omega, \vartheta, \lambda)}\left(H_{L}^{(\omega, \vartheta, \lambda)}\right)\right\},
$$

while the electromagnetic potential energy (increment) $\mathbf{P}^{(\omega, \mathbf{A})} \equiv \mathbf{P}^{(\beta, \omega, \vartheta, \lambda, \mathbf{A})}$ is $\mathbf{P}^{(\omega, \mathbf{A})}(t):=\rho_{t}^{(\beta, \omega, \vartheta, \lambda, \mathbf{A})}\left(W_{t}^{(\omega, \vartheta, \mathbf{A})}\right)=\rho_{t}^{(\beta, \omega, \vartheta, \lambda, \mathbf{A})}\left(W_{t}^{(\omega, \vartheta, \mathbf{A})}\right)-\varrho^{(\beta, \omega, \vartheta, \lambda)}\left(W_{t_{0}}^{(\omega, \vartheta, \mathbf{A})}\right)$ for any $\beta \in \mathbb{R}^{+}, \omega \in \Omega, \vartheta, \lambda \in \mathbb{R}_{0}^{+}, \mathbf{A} \in \mathbf{C}_{0}^{\infty}$ and $t \in \mathbb{R}$.

Then, by using Lieb-Robinson bounds for multi-commutators [BP3, Theorems 3.8-3.9] of order three in a decisive way, we extend [BPH1, Theorems 3.2, 5.8] to fermion systems with short-range interactions:

\section{Theorem 3.2 (Heat, electromagnetic work and 1st law of thermodynamics)}

Assume (10)-(11) and (24). Let $\beta \in \mathbb{R}^{+}, \omega \in \Omega, \vartheta, \lambda \in \mathbb{R}_{0}^{+}$and $\mathbf{A} \in \mathbf{C}_{0}^{\infty}$.

(i) 1st law of thermodynamics: For any $B \in \mathcal{U}_{0}$ and $t \in \mathbb{R}$,

$$
\delta^{(\omega, \vartheta, \lambda)} \circ \tau_{t}^{(\omega, \vartheta, \lambda)}(B)=\lim _{L \rightarrow \infty} i\left[H_{L}^{(\omega, \vartheta, \lambda)}, \tau_{t}^{(\omega, \vartheta, \lambda)}(B)\right] \in \mathcal{U}
$$


and, for any $t \geq t_{0}$,

$$
\mathbf{Q}^{(\omega, \mathbf{A})}(t)=\mathbf{S}^{(\omega, \mathbf{A})}(t) \in \mathbb{R}_{0}^{+} .
$$

(ii) Total energy increment and electromagnetic work: For any $t \geq t_{0}$,

$$
\mathbf{S}^{(\omega, \mathbf{A})}(t)+\mathbf{P}^{(\omega, \mathbf{A})}(t)=\int_{t_{0}}^{t} \rho_{s}^{(\beta, \omega, \vartheta, \lambda, \mathbf{A})}\left(\partial_{s} W_{s}^{(\omega, \vartheta, \mathbf{A})}\right) \mathrm{d} s .
$$

In particular, the maps $\mathbf{Q}^{(\omega, \mathbf{A})}$ and $\mathbf{S}^{(\omega, \mathbf{A})}$ respectively defined by Definition 3.1 and (26) take always positive and finite values for all times.

Proof: $\quad$ To simplify, we fix $\beta \in \mathbb{R}^{+}, \omega \in \Omega, \vartheta, \lambda \in \mathbb{R}_{0}^{+}, \mathbf{A} \in \mathbf{C}_{0}^{\infty}$ and use the notation $\delta \equiv \delta^{(\omega, \vartheta, \lambda)}, \tau_{t} \equiv \tau_{t}^{(\omega, \vartheta, \lambda)}, \tau_{t}^{(L)} \equiv \tau_{t}^{(\omega, \vartheta, \lambda, L)}$, and

$$
\delta^{(L)}(B):=i\left[H_{L}^{(\omega, \vartheta, \lambda)}, B\right], \quad B \in \mathcal{U} .
$$

See (15) and Theorem 2.1.

(i) By using the stationarity of the KMS state $\varrho^{(\beta, \omega, \vartheta, \lambda)}$ w.r.t. the unperturbed dynamics as well as Theorem 2.2 (iii), we infer from (23) that, for any $t \geq t_{0}$,

$$
\rho_{t}^{(\beta, \omega, \vartheta, \lambda, \mathbf{A})}(B)=\varrho^{(\beta, \omega, \vartheta, \lambda)}\left(\mathfrak{U}_{t, t_{0}}^{*} B \mathfrak{U}_{t, t_{0}}\right), \quad B \in \mathcal{U} .
$$

Recall that $\left\{\mathfrak{U}_{t, t_{0}}\right\}_{t \geq t_{0}} \subset \operatorname{Dom}(\delta)$, see (19)-(20). Assume that

$$
\delta\left(\mathfrak{U}_{t, t_{0}}\right)=\lim _{L \rightarrow \infty} \delta^{(L)}\left(\mathfrak{U}_{t, t_{0}}\right) \in \mathcal{U} .
$$

Then, using this together with (29) and the continuity of states, one gets

$$
\mathbf{S}^{(\omega, \mathbf{A})}(t)=-i \varrho^{(\beta, \omega, \vartheta, \lambda)}\left(\mathfrak{U}_{t, t_{0}}^{*} \delta\left(\mathfrak{U}_{t, t_{0}}\right)\right) \in \mathbb{R},
$$

similar to [BPH1, Theorem 5.5]. Since, by definition, $\varrho^{(\beta, \omega, \vartheta, \lambda)}$ is a $\left(\tau^{(\omega, \vartheta, \lambda)}, \beta\right)-$ KMS state, [JP, Theorem 1.1] implies from (31) that, for any $t \geq t_{0}$,

$$
\mathbf{S}^{(\omega, \mathbf{A})}(t)=\mathbf{Q}^{(\omega, \mathbf{A})}(t) \geq 0 .
$$

In other words, we obtain the 1st law of thermodynamics in the same way one gets [BPH1, Theorems 5.3, 5.5, Corollaries 5.6-5.7], provided (30) holds true.

Equation (30) is not trivial at all in the general case. Indeed, as one can see from (20), $\mathfrak{U}_{t, t_{0}} \in \operatorname{Dom}(\delta)$ is generally not in $\mathcal{U}_{0}$ and, by Theorem 2.1 (ii), we only know so far that

$$
\delta(B)=\lim _{L \rightarrow \infty} \delta^{(L)}(B) \in \mathcal{U}, \quad B \in \mathcal{U}_{0},
$$


$\mathcal{U}_{0}$ being a core for $\delta$. By using (24), Equation (32) can however be extended to all elements of $\tau_{t}\left(\mathcal{U}_{0}\right)$ for any $t \in \mathbb{R}$, as explained below.

Indeed, using in the autonomous case very similar arguments to [BP3, Eqs. (120)-(128)] we can apply Lieb-Robinson bounds for multi-commutators of order three, i.e., [BP3, Theorems 3.8-3.9], together with the proof of [BP3, Lemma 3.2] (in particular [BP3, Eqs. (23)-(27)]) to deduce that $\left\{\delta \circ \tau_{t}^{(L)}(B)\right\}_{L \in \mathbb{R}^{+}}$is a Cauchy net within the complete space $\mathcal{U}$ for any $B \in \mathcal{U}_{0}$. These arguments are rather long to write down and there is no reason to reproduce them here again in detail. Note only that they can be applied because of Condition (24) with $\varsigma>2 d$. These inequalities are used to get the estimate [BP3, Eq. (127)] in the non-autonomous case.

By Theorem 2.1 (i), $\left\{\tau_{t}^{(L)}\right\}_{L \in \mathbb{R}^{+}}$converges strongly to $\tau_{t}$ for every $t \in \mathbb{R}$, while Theorem 2.1 (ii) says that $\delta$ is a closed operator. Therefore, for any $B \in \mathcal{U}_{0}$ and $t \in \mathbb{R}, \tau_{t}(B) \in \operatorname{Dom}(\delta)$ and the family $\left\{\delta \circ \tau_{t}^{(L)}(B)\right\}_{L \in \mathbb{R}^{+}}$converges to $\delta \circ \tau_{t}(B)$, i.e., by (32),

$$
\delta \circ \tau_{t}(B)=\lim _{L_{2} \rightarrow \infty} \lim _{L_{1} \rightarrow \infty} \delta^{\left(L_{1}\right)} \circ \tau_{t}^{\left(L_{2}\right)}(B) .
$$

Now, we combine Theorem 2.1 and the Lieb-Robinson bounds [BP3, Theorem 3.1] for the finite volume dynamics with (10)-(11), (32) and Lebesgue's dominated convergence theorem to compute from (33) that

$$
\delta \circ \tau_{t}(B)=i \sum_{x, y \in \mathfrak{L}}\left\langle\mathfrak{e}_{x},\left(\Delta_{\omega, \vartheta}+\lambda V_{\omega}\right) \mathfrak{e}_{y}\right\rangle\left[a_{x}^{*} a_{y}, \tau_{t}(B)\right]+i \sum_{\Lambda \in \mathcal{P}_{f}(\mathfrak{L})}\left[\Psi_{\Lambda}^{\mathrm{IP}}, \tau_{t}(B)\right]
$$

for any $B \in \mathcal{U}_{0}$ and $t \in \mathbb{R}$. For more details, compare for instance with [BP3, Eqs. (38)-(40)]. By using Lieb-Robinson bounds for the infinite-volume dynamics (Theorem 2.1 (iii)) together with (14) and (28), we arrive at

$$
\delta \circ \tau_{t}(B)=\lim _{L \rightarrow \infty} \delta^{(L)} \circ \tau_{t}(B) \in \mathcal{U}, \quad B \in \mathcal{U}_{0}, t \in \mathbb{R}
$$

which is an extension of (32) to all elements of $\tau_{t}\left(\mathcal{U}_{0}\right)$ for any $t \in \mathbb{R}$.

Now, to prove (30) from (20) we use (34) together with the fact that $\delta$ is a symmetric derivation. Indeed, (20) together with the equation

$$
\delta\left(B_{1} B_{2}\right)=\delta\left(B_{1}\right) B_{2}+B_{1} \delta\left(B_{2}\right), \quad B_{1}, B_{2} \in \operatorname{Dom}(\delta),
$$


yields

$$
\begin{gathered}
\delta\left(\mathfrak{U}_{t, t_{0}}\right)=-\sum_{k \in \mathbb{N}}(-i)^{k+1} \int_{s}^{t} \mathrm{~d} s_{1} \cdots \int_{s}^{s_{k-1}} \mathrm{~d} s_{k} \sum_{j=1}^{k} \tau_{s_{1}-t}\left(W_{s_{1}}^{(\omega, \vartheta, \mathbf{A})}\right) \\
\cdots \delta\left(\tau_{s_{j}-t}\left(W_{s_{j}}^{(\omega, \vartheta, \mathbf{A})}\right)\right) \cdots \tau_{s_{k}-t}\left(W_{s_{k}}^{(\omega, \vartheta, \mathbf{A})}\right)
\end{gathered}
$$

for any $t \geq t_{0}$. Observe that a few simple estimates on

$$
\delta\left(\tau_{s_{j}-t}\left(W_{s_{j}}^{(\omega, \vartheta, \mathbf{A})}\right)\right)=\tau_{s_{j}-t}\left(\delta\left(W_{s_{j}}^{(\omega, \vartheta, \mathbf{A})}\right)\right)
$$

are also needed to obtain (35). We omit the details. Combined with (10)-(11), (34), Theorem 2.1 (iii) and Lebesgue's dominated convergence theorem, Equation (35) implies (30). Note that $\mathbf{A} \in \mathbf{C}_{0}^{\infty}$ and $W_{t}^{(\omega, \vartheta, \mathbf{A})} \in \mathcal{U}_{0}$ for any $t \geq t_{0}$, by (18). The proof of Assertion (i) is thus completed.

(ii) We omit the details since it is an extension of [BR2, Lemma 5.4.27.] to unbounded symmetric derivations $\delta^{(\omega, \vartheta, \lambda)}$ already done in the proof of [BPH1, Theorem 5.8].

\section{Remark 3.3 (1st law of thermodynamics)}

If Conditions (10)-(11) and (24) are satisfied then the 1st law of thermodynamics, corresponding to Theorem 3.2 (i) in our specific case, holds true for any interaction $\Psi \in \mathcal{W}$ and static potential $\mathbf{V}$ at thermal equilibrium. By static potential, we mean here a collection $\mathbf{V}:=\left\{\mathbf{V}_{\{x\}}\right\}_{x \in \mathfrak{L}}$ of even and self-adjoint elements such that $\mathbf{V}_{\{x\}}=\mathbf{V}_{\{x\}}^{*} \in \mathcal{U}^{+} \cap \mathcal{U}_{\{x\}}$ for all $x \in \mathfrak{L}$. See [BP3] for the general setting. This observation is a nontrivial consequence of Lieb-Robinson bounds for multi-commutators of order three, see [BP3, Theorems 3.8-3.9].

[BPH1, Theorem 3.4] describes the behavior of the heat production at weak electromagnetic fields of free lattice fermions in thermal equilibrium. In the following we extend this result to fermion systems with short-range interactions: For any $l \in \mathbb{R}^{+}$and $\mathbf{A} \in \mathbf{C}_{0}^{\infty}$, we consider the space-rescaled vector potential

$$
\mathbf{A}_{l}(t, x):=\mathbf{A}\left(t, l^{-1} x\right), \quad t \in \mathbb{R}, x \in \mathbb{R}^{d} .
$$

Since Ohm's law is a linear response to electric fields, we also rescale the strength of the electromagnetic potential $\mathbf{A}_{l}$ by a real parameter $\eta \in \mathbb{R}$ and study the behavior of the heat production in the limit $\eta \rightarrow 0$. 
Conditions (21)-(22) and [BP3, Eq. (155)], that is, for all parameters $\eta, \eta_{0} \in$ $\mathbb{R}$

$$
\sup _{x, y \in \mathfrak{L}} \sup _{t \in \mathbb{R}}\left|\mathbf{w}_{x, y}(\eta, t)-\mathbf{w}_{x, y}\left(\eta_{0}, t\right)\right| \leq D\left|\eta-\eta_{0}\right|
$$

are satisfied by the perturbation $W_{t}^{(\omega, \vartheta, \mathbf{A})}$. By Theorem 3.2 (i), we can apply either [BP3, Theorem 4.8] or [BP3, Theorem 4.9], depending on the space decay of interparticle interaction $\Psi^{\mathrm{IP}}$, to obtain the behavior of the heat production $\mathbf{Q}^{\left(\omega, \eta \mathbf{A}_{l}\right)}$ w.r.t. $\eta, l \in \mathbb{R}^{+}$. The interaction $\Phi$ appearing in [BP3, Theorem 4.8] and [BP3, Theorem 4.9] is in this case the sum of the interaction $\Psi^{\mathrm{IP}}$ with an element from $\mathcal{W}$ taking into account the hoppings of particles and external static potential which are encoded via

$$
\omega=\left(\omega_{1}, \omega_{2}\right) \in \Omega:=[-1,1]^{\mathfrak{L}} \times \mathbb{D}^{\mathfrak{b}},
$$

where we recall that $\mathbb{D}:=\{z \in \mathbb{C}:|z| \leq 1\}$. Note that the map

$$
\eta \mapsto W_{s}^{\left(\omega, \vartheta, \eta \mathbf{A}_{l}\right)} \in \mathcal{U}
$$

is real analytic and the conditions of [BP3, Theorem 4.9] are satisfied if the function $\mathbf{F}$ defining the norm of the space $\mathcal{W}$ of interactions decays sufficiently fast at large arguments. Theorem 3.2 (i) combined with [BP3, Theorem 4.9] implies that $\eta \mapsto \mathbf{Q}^{\left(\omega, \eta \mathbf{A}_{l}\right)}$ is a Gevrey function on $\mathbb{R}$ in this specific case.

With the arguments using [BP3, Theorems 4.8-4.9] discussed above, [BPH1, Theorem 3.4] can straightforwardly be extended to fermion systems with shortrange interactions. Note, moreover, that [BP3, Theorems 4.8-4.9] also make possible the study of non-quadratic (resp. non-linear) corrections to Joule's law (resp. Ohm's law). The minimal requirement to study the linear response to electric fields is some sufficiently fast polynomial decay of interparticle interactions, that is, Condition (24).

\subsection{Charge Transport Coefficients}

Fix $\omega \in \Omega, \vartheta, \lambda \in \mathbb{R}_{0}^{+}$. The paramagnetic current observables is defined by

$$
I_{\mathbf{x}}^{(\omega, \vartheta)}:=-2 \operatorname{Im}\left(\left\langle\mathfrak{e}_{x^{(1)}}, \Delta_{\omega, \vartheta} \mathfrak{e}_{x^{(2)}}\right\rangle a_{x^{(1)}}^{*} a_{x^{(2)}}\right), \quad \mathbf{x}:=\left(x^{(1)}, x^{(2)}\right) \in \mathfrak{L}^{2} .
$$

Indeed, $I_{\mathrm{x}}^{(\omega, \vartheta)} \in \mathcal{U}_{0}$ is seen as a current observable because, by Theorem 2.1 (ii), it satisfies the discrete continuity equation

$$
\partial_{t}\left(\tau_{t}^{(\omega, \vartheta, \lambda)}\left(a_{x}^{*} a_{x}\right)\right)=\tau_{t}^{(\omega, \vartheta, \lambda)}\left(-\sum_{y \in \mathfrak{L},|x-y|=1} I_{(x, y)}^{(\omega, \vartheta)}+i \sum_{\Lambda \in \mathcal{P}_{f}(\mathfrak{L})}\left[\Psi_{\Lambda}^{\mathrm{IP}}, a_{x}^{*} a_{x}\right]\right)
$$


for any $x \in \mathfrak{L}$ and $t \in \mathbb{R}$.

For short-range interactions satisfying

$$
\sum_{\Lambda \in \mathcal{P}_{f}(\mathfrak{L})}\left[\Psi_{\Lambda}^{\mathrm{IP}}, a_{x}^{*} a_{x}\right]=0, \quad x \in \mathfrak{L},
$$

i.e., $\Psi^{\mathrm{IP}}$ is invariant under local gauge transformations, $I_{\mathrm{x}}^{(\omega, \vartheta)}$ is thus the observable related, in absence of external electromagnetic potentials, to the flow of particles from the lattice site $x^{(1)}$ to the lattice site $x^{(2)}$ or the current from $x^{(2)}$ to $x^{(1)}$. [Positively charged particles can of course be treated in the same way.]

We also define

$$
P_{\mathbf{x}}^{(\omega, \vartheta)}:=2 \operatorname{Re}\left(\left\langle\mathfrak{e}_{x^{(1)}}, \Delta_{\omega, \vartheta} \mathfrak{e}_{x^{(2)}}\right\rangle a_{x^{(1)}}^{*} a_{x^{(2)}}\right), \quad \mathbf{x}:=\left(x^{(1)}, x^{(2)}\right) \in \mathfrak{L}^{2} .
$$

For real-valued $\omega_{2}\left(x^{(1)}, x^{(2)}\right)$, this self-adjoint element of $\mathcal{U}_{0}$ is proportional to the second-quantization of the adjacency matrix of the oriented graph containing exactly the oriented bonds $\left(x^{(2)}, x^{(1)}\right)$ and $\left(x^{(1)}, x^{(2)}\right)$.

Now, for any $\beta \in \mathbb{R}^{+}, \omega \in \Omega$ and $\vartheta, \lambda \in \mathbb{R}_{0}^{+}$, we introduce two important functions associated with the above defined observables $I_{\mathbf{x}}^{(\omega, \vartheta)}$ and $P_{\mathbf{x}}^{(\omega, \vartheta)}$ :

(p) The paramagnetic transport coefficient $\sigma_{\mathrm{p}}^{(\omega)} \equiv \sigma_{\mathrm{p}}^{(\beta, \omega, \vartheta, \lambda)}$ is defined, for any $\mathbf{x}, \mathbf{y} \in \mathfrak{L}^{2}$ and $t \in \mathbb{R}$, by

$$
\sigma_{\mathrm{p}}^{(\omega)}(\mathbf{x}, \mathbf{y}, t):=\int_{0}^{t} \varrho^{(\beta, \omega, \vartheta, \lambda)}\left(i\left[I_{\mathbf{y}}^{(\omega, \vartheta)}, \tau_{s}^{(\omega, \vartheta, \lambda)}\left(I_{\mathbf{x}}^{(\omega, \vartheta)}\right)\right]\right) \mathrm{d} s .
$$

(d) The diamagnetic transport coefficient $\sigma_{\mathrm{d}}^{(\omega)} \equiv \sigma_{\mathrm{d}}^{(\beta, \omega, \vartheta, \lambda)}$ is defined by

$$
\sigma_{\mathrm{d}}^{(\omega)}(\mathbf{x}):=\varrho^{(\beta, \omega, \vartheta, \lambda)}\left(P_{\mathbf{x}}^{(\omega, \vartheta)}\right), \quad \mathbf{x} \in \mathfrak{L}^{2} .
$$

As explained in [BPH2, Section 3.3], $\sigma_{\mathrm{p}}^{(\omega)}$ can be associated with a "quantum current viscosity", while $\sigma_{\mathrm{d}}^{(\omega)}$ is related to the ballistic motion of charged particles within electric fields.

For large samples (i.e., large $l \in \mathbb{R}^{+}$), we then define the space-averaged paramagnetic transport coefficient

$$
t \mapsto \Xi_{\mathrm{p}, l}^{(\omega)}(t) \equiv \Xi_{\mathrm{p}, l}^{(\beta, \omega, \vartheta, \lambda)}(t) \in \mathcal{B}\left(\mathbb{R}^{d}\right)
$$


w.r.t. the canonical orthonormal basis $\left\{e_{k}\right\}_{k=1}^{d}$ of the Euclidian space $\mathbb{R}^{d}$ by

$$
\left\{\Xi_{\mathrm{p}, l}^{(\omega)}(t)\right\}_{k, q}:=\frac{1}{\left|\Lambda_{l}\right|} \sum_{x, y \in \Lambda_{l}} \sigma_{\mathrm{p}}^{(\omega)}\left(x+e_{k}, x, y+e_{q}, y, t\right)
$$

for any $l, \beta \in \mathbb{R}^{+}, \omega \in \Omega, \vartheta, \lambda \in \mathbb{R}_{0}^{+}, k, q \in\{1, \ldots, d\}$ and $t \in \mathbb{R}$. The spaceaveraged diamagnetic transport coefficient

$$
\Xi_{\mathrm{d}, l}^{(\omega)} \equiv \Xi_{\mathrm{d}, l}^{(\beta, \omega, \vartheta, \lambda)} \in \mathcal{B}\left(\mathbb{R}^{d}\right)
$$

corresponds to the diagonal matrix defined by

$$
\left\{\Xi_{\mathrm{d}, l}^{(\omega)}\right\}_{k, q}:=\frac{\delta_{k, q}}{\left|\Lambda_{l}\right|} \sum_{x \in \Lambda_{l}} \sigma_{\mathrm{d}}^{(\omega)}\left(x+e_{k}, x\right) \in[-2(\vartheta+1), 2(\vartheta+1)] .
$$

Both coefficients are typically the paramagnetic and diamagnetic (in-phase) conductivities one experimentally measures in all space directions.

The main properties of the paramagnetic transport coefficient $\Xi_{\mathrm{p}, l}^{(\omega)}$ are given in the next theorem. To state it, we introduce some notation: $\mathcal{B}_{+}\left(\mathbb{R}^{d}\right) \subset \mathcal{B}\left(\mathbb{R}^{d}\right)$ stands for the set of positive linear operators on $\mathbb{R}^{d}$ (i.e., symmetric operators w.r.t. to the canonical scalar product of $\mathbb{R}^{d}$ with non-negative eigenvalues). For any $\mathcal{B}\left(\mathbb{R}^{d}\right)$-valued measure $\mu$ on $\mathbb{R},\|\mu\|_{\text {op }}$ denotes the positive measure on $\mathbb{R}$ defined, for any Borel set $\mathcal{X}$, by

$$
\|\mu\|_{\text {op }}(\mathcal{X}):=\sup \left\{\sum_{i \in I}\left\|\mu\left(\mathcal{X}_{i}\right)\right\|_{\text {op }}:\left\{\mathcal{X}_{i}\right\}_{i \in I} \text { is a finite Borel partition of } \mathcal{X}\right\} .
$$

We say that $\mu$ is symmetric if $\mu(\mathcal{X})=\mu(-\mathcal{X})$ for any Borel set $\mathcal{X} \subset \mathbb{R}$. Additionally, for any $\Theta \in \mathcal{B}\left(\mathbb{R}^{d}\right)$, define its symmetric and antisymmetric parts (w.r.t. to the canonical scalar product of $\mathbb{R}$ ) respectively by

$$
[\Theta]_{+}:=\frac{1}{2}\left(\Theta+\Theta^{\mathrm{t}}\right) \quad \text { and } \quad[\Theta]_{-}:=\frac{1}{2}\left(\Theta-\Theta^{\mathrm{t}}\right) .
$$

Here, $\Theta^{t} \in \mathcal{B}\left(\mathbb{R}^{d}\right)$ stands for the transpose w.r.t. the canonical scalar product of $\mathbb{R}$ of the operator $\Theta \in \mathcal{B}\left(\mathbb{R}^{d}\right)$. With these definitions we have the following assertion:

Theorem 3.4 (Microscopic paramagnetic conductivity measures)

Assume (10)-(11). For any $l, \beta \in \mathbb{R}^{+}, \omega \in \Omega$ and $\vartheta, \lambda \in \mathbb{R}_{0}^{+}$, there exists a 
(generally non-zero) symmetric $\mathcal{B}_{+}\left(\mathbb{R}^{d}\right)$-valued measure $\mu_{\mathrm{p}, l}^{(\omega)} \equiv \mu_{\mathrm{p}, l}^{(\beta, \omega, \vartheta, \lambda)}$ on $\mathbb{R}$ such that

$$
\int_{\mathbb{R}}(1+|\nu|)\left\|\mu_{\mathrm{p}, l}^{(\omega)}\right\|_{\mathrm{op}}(\mathrm{d} \nu)<\infty
$$

and

$$
\left[\Xi_{\mathrm{p}, l}^{(\omega)}(t)\right]_{+}=\int_{\mathbb{R}}(\cos (t \nu)-1) \mu_{\mathrm{p}, l}^{(\omega)}(\mathrm{d} \nu), \quad t \in \mathbb{R}
$$

Proof: The assertion is proven by using the Duhamel two-point function exactly as in [BPH2, Section 5.1.2], taking into account only the symmetric part of $\Xi_{\mathrm{p}, l}^{(\omega)}$ and using the property

$$
\Xi_{\mathrm{p}, l}^{(\omega)}(-t)=\left[\Xi_{\mathrm{p}, l}^{(\omega)}(t)\right]^{\mathrm{t}}, \quad t \in \mathbb{R} .
$$

Indeed, the model considered in [BPH2] is time-reversal invariant. In this case, one has $\left[\Xi_{\mathrm{p}, l}^{(\omega)}(t)\right]_{+}=\Xi_{\mathrm{p}, l}^{(\omega)}(t)$. The more general model studied here does not have this symmetry for non-real hopping amplitudes and/or for interparticle interactions which are not invariant w.r.t. to time-reversal. Another difference as compared to systems of non-interacting fermions of [BPH2] concerns [BPH2, Lemma 5.10] which cannot be a priori extended to the interacting case. It follows that (47) may not be uniformly bounded w.r.t. $l, \beta \in \mathbb{R}^{+}, \omega \in \Omega, \lambda \in \mathbb{R}_{0}^{+}$.

One can use the Duhamel scalar product to represent (up to a real constant) the entries of $\Xi_{\mathrm{p}, l}^{(\omega)}(t)$ w.r.t. the canonical basis of $\mathbb{R}^{d}$ as matrix elements of some unitary one-parameter group $\{U(t)\}_{t \in \mathbb{R}}$ on a Hilbert space. Then, one gets a similar expression as in Theorem 3.4 for the full coefficient $\Xi_{\mathrm{p}, l}^{(\omega)}$ in terms of a measure. However, if $\left[\Xi_{\mathrm{p}, l}^{(\omega)}(t)\right]_{-}$does not vanish for some time $t$, then the corresponding measure is not anymore symmetric w.r.t. $\nu$ and it does not take values in the set of symmetric matrices, in general. Moreover, the symmetric component $\left[\Xi_{\mathrm{p}, l}^{(\omega)}(t)\right]_{+}$ of $\Xi_{\mathrm{p}, l}^{(\omega)}(t)$ corresponds to the real part of $U(t)$ and the antisymmetric component $\left[\Xi_{\mathrm{p}, l}^{(\omega)}(t)\right]_{-}$to the imaginary part of the same unitary. This is a consequence of (48).

For any $\vec{w} \in \mathbb{R}^{d}$ and $t \in \mathbb{R},\left[\Xi_{\mathrm{p}, l}^{(\omega)}(t)\right]_{-} \vec{w} \in \mathbb{R}^{d}$ and $\vec{w}$ are orthogonal (w.r.t. the canonical scalar product of $\mathbb{R}^{d}$ ). So, $\left[\Xi_{\mathrm{p}, l}^{(\omega)}\right]_{-} \neq 0$ can be physically seen as the presence of effective magnetic fields in the system, assuming that $\Xi_{\mathrm{p}, l}^{(\omega)}$ correctly describes the paramagnetic conductivity. As mentioned in the proof of the above theorem, $\left[\Xi_{\mathrm{p}, l}^{(\omega)}\right]_{-}=0$ whenever the system is invariant w.r.t. time-reversal, but non-vanishing magnetic fields break this symmetry. The conductivity measure 
describes the heat production in the presence of electric fields which are spacehomogeneous. Since currents which are orthogonal to the applied fields are physically not expected to produce heat, it is not astonishing that $\mu_{\mathrm{p}, l}^{(\omega)}$ only depends on the symmetric part of the conductivity $\Xi_{\mathrm{p}, l}^{(\omega)}$.

Corollary 3.5 (Properties of $\left[\Xi_{\mathrm{p}, l}^{(\omega)}\right]_{+}$)

Assume (10)-(11). For $l, \beta \in \mathbb{R}^{+}, \omega \in \Omega$ and $\vartheta, \lambda \in \mathbb{R}_{0}^{+}$, the symmetric part of $\Xi_{\mathrm{p}, l}^{(\omega)}$ has the following properties:

(i) Time-reversal symmetry of $\left[\Xi_{\mathrm{p}, l}^{(\omega)}\right]_{+}:\left[\Xi_{\mathrm{p}, l}^{(\omega)}(0)\right]_{+}=0$ and

$$
\left[\Xi_{\mathrm{p}, l}^{(\omega)}(-t)\right]_{+}=\left[\Xi_{\mathrm{p}, l}^{(\omega)}(t)\right]_{+}, \quad t \in \mathbb{R} .
$$

(ii) Negativity of $\left[\Xi_{\mathrm{p}, l}^{(\omega)}\right]_{+}$:

$$
-\left[\Xi_{\mathrm{p}, l}^{(\omega)}(t)\right]_{+} \in \mathcal{B}_{+}\left(\mathbb{R}^{d}\right), \quad t \in \mathbb{R} .
$$

(iii) Cesàro mean of $\left[\Xi_{\mathrm{p}, l}^{(\omega)}\right]_{+}$:

$$
\lim _{t \rightarrow \infty} \frac{1}{t} \int_{0}^{t}\left[\Xi_{\mathrm{p}, l}^{(\omega)}(s)\right]_{+} \mathrm{d} s=-\mu_{\mathrm{p}, l}^{(\omega)}(\mathbb{R} \backslash\{0\}) .
$$

Proof: (i)-(iii) are direct consequences of Theorem 3.4 and Lebesgue's dominated convergence theorem.

Recall that (47) is a priori not uniformly bounded w.r.t. the parameters $l, \beta \in$ $\mathbb{R}^{+}, \omega \in \Omega, \lambda \in \mathbb{R}_{0}^{+}$as in [BPH2, Theorem 3.1]. In particular, the family

$$
\left\{\Xi_{\mathrm{p}, l}^{(\beta, \omega, \vartheta, \lambda)}\right\}_{l, \beta \in \mathbb{R}^{+}, \omega \in \Omega, \lambda \in \mathbb{R}_{0}^{+}}
$$

of maps from $\mathbb{R}$ to $\mathcal{B}\left(\mathbb{R}^{d}\right)$ may not be equicontinuous. Compare with [BPH2, Corollary 3.2 (iv)]. We deduce this property for times on compacta from LiebRobinson bounds:

\section{Theorem 3.6 (Uniform boundedness and equicontinuity properties)}

Assume (10)-(11). Let $\vartheta_{0}, \mathrm{~T} \in \mathbb{R}^{+}$. Then, for any $l, \beta \in \mathbb{R}^{+}, \omega \in \Omega, \vartheta \in\left[0, \vartheta_{0}\right]$, $\lambda \in \mathbb{R}_{0}^{+}$and $t_{1}, t_{2} \in[-\mathrm{T}, \mathrm{T}]$,

$\left\|\Xi_{\mathrm{p}, l}^{(\omega)}\left(t_{1}\right)-\Xi_{\mathrm{p}, l}^{(\omega)}\left(t_{2}\right)\right\|_{\max } \leq 32\left(1+\vartheta_{0}\right)^{2}\left(\mathbf{D}^{-1}\|\mathbf{F}\|_{1, \mathfrak{L}} \mathrm{e}^{4 \mathbf{D}|\mathrm{T}| D_{\vartheta_{0}}}+1\right)\left|t_{2}-t_{1}\right|$, where $\|\cdot\|_{\max }$ is the max norm of matrices. 
Proof: $\quad$ It is a direct application of Theorem 2.1 (iii).

As in [BPH2, Section 5.1.2] the $\mathcal{B}_{+}\left(\mathbb{R}^{d}\right)$-valued measures $\mu_{\mathrm{p}, l}^{(\omega)}$ can be represented in terms of the spectral measure of an explicit self-adjoint operator w.r.t. explicitly given vectors. The constant $\mu_{\mathrm{p}, l}^{(\omega)}(\mathbb{R} \backslash\{0\})$ is the so-called static admittance of linear response theory. Moreover, if $\Xi_{\mathrm{d}, l}^{(\omega)}$ is non-degenerated then one can construct $\mu_{\mathrm{p}, l}^{(\omega)}$ from the space-averaged quantum current viscosity

$$
\mathbf{V}_{l}^{(\omega)}(t) \equiv \mathbf{V}_{l}^{(\beta, \omega, \vartheta, \lambda)}(t):=\left(\Xi_{\mathrm{d}, l}^{(\omega)}\right)^{-1} \partial_{t}\left[\Xi_{\mathrm{p}, l}^{(\omega)}(t)\right]_{+} \in \mathcal{B}\left(\mathbb{R}^{d}\right)
$$

for any $l, \beta \in \mathbb{R}^{+}, \omega \in \Omega, \vartheta, \lambda \in \mathbb{R}_{0}^{+}$and $t \in \mathbb{R}$. Indeed, $\mu_{\mathrm{p}, l}^{(\omega)}$ is the boundary value of the (imaginary part of the) Laplace-Fourier transform of $\Xi_{\mathrm{d}, l}^{(\omega)} \mathbf{V}_{l}^{(\omega)}$. For more details, see [BPH2, Sections 3.3, 5.1.2]. As compared to [BPH2, Sections $3.3,5.1 .2]$, note that $\Xi_{\mathrm{p}, l}^{(\omega)}$ is replaced in (49) with its symmetric part.

\subsection{Microscopic Ohm's Law}

The diamagnetic current observable $\mathrm{I}_{\mathbf{x}}^{(\omega, \vartheta, \mathbf{A})}$ is defined, for any $\omega \in \Omega, \vartheta \in \mathbb{R}_{0}^{+}$, $\mathbf{A} \in \mathbf{C}_{0}^{\infty}, t \geq t_{0}$ and $\mathbf{x}:=\left(x^{(1)}, x^{(2)}\right) \in \mathfrak{L}^{2}$, by

$$
\begin{aligned}
\mathrm{I}_{\mathbf{x}}^{(\omega, \vartheta, \mathbf{A})} \equiv \mathrm{I}_{\mathbf{x}}^{(\omega, \vartheta, \mathbf{A}(t, \cdot))}:=-2 \operatorname{Im}\left(\left(\mathrm{e}^{-i \int_{0}^{1}\left[\mathbf{A}\left(t, \alpha x^{(2)}+(1-\alpha) x^{(1)}\right)\right]\left(x^{(2)}-x^{(1)}\right) \mathrm{d} \alpha}-1\right)\right. \\
\left.\times\left\langle\mathfrak{e}_{x^{(1)}}, \Delta_{\omega, \vartheta} \mathfrak{e}_{x^{(2)}}\right\rangle a_{x^{(1)}}^{*} a_{x^{(2)}}\right) .
\end{aligned}
$$

It corresponds to a correction to the current $I_{\mathrm{x}}^{(\omega, \vartheta)}$ defined above engendered by the presence of an external electromagnetic potential. See [BPH2, Section 3.1] for more details.

Like in [BPH2, Section 3], w.l.o.g. we only consider space-homogeneous (though time-dependent) electric fields in the box $\Lambda_{l}$ defined by (6) for $l \in \mathbb{R}^{+}$. More precisely, let $\vec{w}:=\left(w_{1}, \ldots, w_{d}\right) \in \mathbb{R}^{d}$ be any (normalized w.r.t. the usual Euclidian norm) vector, $\mathcal{A} \in C_{0}^{\infty}(\mathbb{R} ; \mathbb{R})$ and set $\mathcal{E}_{t}:=-\partial_{t} \mathcal{A}_{t}$ for all $t \in \mathbb{R}$. Then, $\overline{\mathbf{A}} \in \mathbf{C}_{0}^{\infty}$ is defined to be the electromagnetic potential such that the electric field equals $\mathcal{E}_{t} \vec{w}$ at time $t \in \mathbb{R}$ for all $x \in[-1,1]^{d}$ and $(0,0, \ldots, 0)$ for $t \in \mathbb{R}$ and $x \notin[-1,1]^{d}$. This choice yields rescaled electromagnetic potentials $\eta \overline{\mathbf{A}}_{l}$ as defined by (36) for $l \in \mathbb{R}^{+}$and $\eta \in \mathbb{R}$.

For any $l, \beta \in \mathbb{R}^{+}, \omega \in \Omega, \vartheta, \lambda \in \mathbb{R}_{0}^{+}, \eta \in \mathbb{R}, \vec{w} \in \mathbb{R}^{d}, \mathcal{A} \in C_{0}^{\infty}(\mathbb{R} ; \mathbb{R})$ and $t \geq t_{0}$, the total current density is the sum of three currents defined from (38) and (50): 
(th) The (thermal) current density

$$
\mathbb{J}_{\mathrm{th}}^{(\omega, l)} \equiv \mathbb{J}_{\mathrm{th}}^{(\beta, \omega, \vartheta, \lambda, l)} \in \mathbb{R}^{d}
$$

at thermal equilibrium inside the box $\Lambda_{l}$ is defined, for any $k \in\{1, \ldots, d\}$, by

$$
\left\{\mathbb{J}_{\mathrm{th}}^{(\omega, l)}\right\}_{k}:=\left|\Lambda_{l}\right|^{-1} \sum_{x \in \Lambda_{l}} \varrho^{(\beta, \omega, \vartheta, \lambda)}\left(I_{\left(x+e_{k}, x\right)}^{(\omega, \vartheta)}\right) .
$$

(p) The paramagnetic current density is the map

$$
t \mapsto \mathbb{J}_{\mathrm{p}}^{\left(\omega, \eta \overline{\mathbf{A}}_{l}\right)}(t) \equiv \mathbb{J}_{\mathrm{p}}^{\left(\beta, \omega, \vartheta, \lambda, \eta \overline{\mathbf{A}}_{l}\right)}(t) \in \mathbb{R}^{d}
$$

defined by the space average of the current increment vector inside the box $\Lambda_{l}$ at times $t \geq t_{0}$, that is for any $k \in\{1, \ldots, d\}$,

$$
\left\{\mathbb{J}_{\mathrm{p}}^{\left(\omega, \eta \overline{\mathbf{A}}_{l}\right)}(t)\right\}_{k}:=\left|\Lambda_{l}\right|^{-1} \sum_{x \in \Lambda_{l}} \rho_{t}^{\left(\beta, \omega, \vartheta, \lambda, \eta \overline{\mathbf{A}}_{l}\right)}\left(I_{\left(x+e_{k}, x\right)}^{(\omega, \vartheta)}\right)-\left\{\mathbb{J}_{\mathrm{th}}^{(\omega, l)}\right\}_{k} .
$$

(d) The diamagnetic (or ballistic) current density

$$
t \mapsto \mathbb{J}_{\mathrm{d}}^{\left(\omega, \eta \overline{\mathbf{A}}_{l}\right)}(t) \equiv \mathbb{J}_{\mathrm{d}}^{\left(\beta, \omega, \vartheta, \lambda, \eta \overline{\mathbf{A}}_{l}\right)}(t) \in \mathbb{R}^{d}
$$

is defined analogously, for any $t \geq t_{0}$ and $k \in\{1, \ldots, d\}$, by

$$
\left\{\mathbb{J}_{\mathrm{d}}^{\left(\omega, \eta \overline{\mathbf{A}}_{l}\right)}(t)\right\}_{k}:=\left|\Lambda_{l}\right|^{-1} \sum_{x \in \Lambda_{l}} \rho_{t}^{\left(\beta, \omega, \vartheta, \lambda, \eta \overline{\mathbf{A}}_{l}\right)}\left(\mathrm{I}_{\left(x+e_{k}, x\right)}^{\left(\omega, \vartheta, \eta \overline{\mathbf{A}}_{l}\right)}\right) .
$$

For more details on the physical interpretations of these currents, we refer to [BPH2, Section 3.4]. We will prove in [BP4] that if $\omega \in \Omega$ is the realization of some ergodic random potential and hopping amplitude then, almost surely, the thermal current density vanishes in the limit $l \rightarrow \infty$.

Similar to [BPH2, Section 3.5], we use the transport coefficients $\Xi_{\mathrm{p}, l}^{(\omega)}(44)$ and $\Xi_{\mathrm{d}, l}^{(\omega)}(45)$ to define two linear response currents

$$
J_{\mathrm{p}, l}^{(\omega, \mathcal{A})} \equiv J_{\mathrm{p}, l}^{(\beta, \omega, \vartheta, \lambda, \vec{w}, \mathcal{A})} \quad \text { and } \quad J_{\mathrm{d}, l}^{(\omega, \mathcal{A})} \equiv J_{\mathrm{d}, l}^{(\beta, \omega, \vartheta, \lambda, \vec{w}, \mathcal{A})}
$$

with values in $\mathbb{R}^{d}$ respectively by

$$
\begin{aligned}
J_{\mathrm{p}, l}^{(\omega, \mathcal{A})}(t) & :=\int_{t_{0}}^{t}\left(\Xi_{\mathrm{p}, l}^{(\omega)}(t-s) \vec{w}\right) \mathcal{E}_{s} \mathrm{~d} s, \quad t \geq t_{0}, \\
J_{\mathrm{d}, l}^{(\omega, \mathcal{A})}(t) & :=\left(\Xi_{\mathrm{d}, l}^{(\omega)} \vec{w}\right) \int_{t_{0}}^{t} \mathcal{E}_{s} \mathrm{~d} s, \quad t \geq t_{0},
\end{aligned}
$$


for any $l, \beta \in \mathbb{R}^{+}, \omega \in \Omega, \vartheta, \lambda \in \mathbb{R}_{0}^{+}, \vec{w} \in \mathbb{R}^{d}$ and $\mathcal{A} \in C_{0}^{\infty}(\mathbb{R} ; \mathbb{R})$. They are the linear responses of the paramagnetic and diamagnetic current densities, respectively:

\section{Theorem 3.7 (Microscopic Ohm's law)}

Assume (10)-(11) and (24). For any $\vartheta_{0} \in \mathbb{R}_{0}^{+}, \mathcal{A} \in C_{0}^{\infty}(\mathbb{R} ; \mathbb{R})$ and $\eta \in \mathbb{R}$,

$\mathbb{J}_{\mathrm{p}}^{\left(\omega, \eta \overline{\mathbf{A}}_{l}\right)}(t)=\eta J_{\mathrm{p}, l}^{(\omega, \mathcal{A})}(t)+\mathcal{O}\left(\eta^{2}\right) \quad$ and $\quad \mathbb{J}_{\mathrm{d}}^{\left(\omega, \eta \overline{\mathbf{A}}_{l}\right)}(t)=\eta J_{\mathrm{d}, l}^{(\omega, \mathcal{A})}(t)+\mathcal{O}\left(\eta^{2}\right)$

uniformly for $l, \beta \in \mathbb{R}^{+}, \omega \in \Omega, \vartheta \in\left[0, \vartheta_{0}\right], \lambda \in \mathbb{R}_{0}^{+}, \vec{w} \in \mathbb{R}^{d}$ (normalized) and $t \geq t_{0}$.

Proof: The assertion directly follows from [BP3, Theorem 4.8], because of Conditions (21)-(22) and (37). We omit the details and refer to [BPH2, Lemmata 5.14-5.15] where similar arguments are used. See also discussions of Section 3.1.

The fact that the asymptotics obtained are uniform w.r.t. $l, \beta \in \mathbb{R}^{+}, \omega \in \Omega$, $\vartheta \in\left[0, \vartheta_{0}\right], \lambda \in \mathbb{R}_{0}^{+}$and $t \geq t_{0}$ is a crucial technical step to get macroscopic Ohm's law when $l \rightarrow \infty$.

Now, all the discussions of [BPH2, Section 3.5] can be reproduced for the interacting fermion system. We refrain from doing this again. We only mention that $\Xi_{\mathrm{p}, l}^{(\omega)}$ and $\Xi_{\mathrm{d}, l}^{(\omega)}$ are the in-phase paramagnetic and diamagnetic (microscopic) conductivity of the fermion system. Indeed, one can deduce from Theorem 3.7 Ohm's law for linear response currents as well as Green-Kubo relations.

Note that this physical interpretation makes only sense when interparticle interactions $\Psi^{\mathrm{IP}}$ are invariant under local gauge transformations, i.e., when they satisfy (40). This condition is obviously satisfied by all density-density interaction like (16). Interparticle interactions related to "hoppings" of particles are not of this type: For example, in microscopic models for superconductors one usually has BCS-type interaction formally like

$$
\sum_{x, y} \gamma(x-y) a_{x, \uparrow}^{*} a_{x, \downarrow}^{*} a_{y, \downarrow} a_{y, \uparrow}
$$

involving fermions of spins $\uparrow$ or $\downarrow$. However, it still possible to handle such models in the setting we describe here, by simply redefining the current observables in such a way that a continuity relation like (39) is fulfilled. See for instance [BP1, Eq. (2.11)] for a concrete example of this procedure. The physical interpretation 
of such a new current is very natural in that case of superconductors: The currents $I_{\mathbf{x}}^{(\omega, \vartheta)}$ as defined above in (38) are related to the flow of single electrons. The correction to it due to terms like (54) in the interaction can be understood as being the contribution of the flow of (Cooper-) paired electrons to the total current. We postpone such a more general study. Note finally that, as in the case of the kinetic term, terms like (54) should also be coupled to electromagnetic fields, similar to Equation (5), since they participate to the total current.

\subsection{Microscopic Joule's Law}

The total energy (25) given by the electromagnetic field to the fermion system is divided in Section 3.1 into two components $\mathbf{S}^{(\omega, \mathbf{A})}(26)$ and $\mathbf{P}^{(\omega, \mathbf{A})}(27)$ that have interesting properties in terms of heat production and potential energy. See for instance Theorem 3.2 and discussions in [BPH1, Section 3.2].

Like in [BPH2, Section 4.3] the total delivered energy can also be naturally divided in two other components with other interesting features, in terms of currents this time. Indeed, for any $\beta \in \mathbb{R}^{+}, \omega \in \Omega, \vartheta, \lambda \in \mathbb{R}_{0}^{+}$and $\mathbf{A} \in \mathbf{C}_{0}^{\infty}$, we define two further energy increments:

(p) The paramagnetic energy increment $\mathfrak{J}_{\mathrm{p}}^{(\omega, \mathbf{A})} \equiv \mathfrak{I}_{\mathrm{p}}^{(\beta, \omega, \vartheta, \lambda, \mathbf{A})}$ is the map from $\mathbb{R}$ to $\mathbb{R}$ defined by

$$
\begin{aligned}
\mathfrak{I}_{\mathrm{p}}^{(\omega, \mathbf{A})}(t):=\lim _{L \rightarrow \infty}\left\{\rho_{t}^{(\beta, \omega, \vartheta, \lambda, \mathbf{A})}\left(H_{L}^{(\omega, \vartheta, \lambda)}+W_{t}^{(\omega, \vartheta, \mathbf{A})}\right)\right. & \\
& \left.-\varrho^{(\beta, \omega, \vartheta, \lambda)}\left(H_{L}^{(\omega, \vartheta, \lambda)}+W_{t}^{(\omega, \vartheta, \mathbf{A})}\right)\right\} .
\end{aligned}
$$

(d) The diamagnetic energy (increment) $\mathfrak{I}_{\mathrm{d}}^{(\omega, \mathbf{A})} \equiv \mathfrak{I}_{\mathrm{d}}^{(\beta, \omega, \vartheta, \lambda, \mathbf{A})}$ is the map from $\mathbb{R}$ to $\mathbb{R}$ defined by

$$
\mathfrak{I}_{\mathrm{d}}^{(\omega, \mathbf{A})}(t):=\varrho^{(\beta, \omega, \vartheta, \lambda)}\left(W_{t}^{(\omega, \vartheta, \mathbf{A})}\right)=\varrho^{(\beta, \omega, \vartheta, \lambda)}\left(W_{t}^{(\omega, \vartheta, \mathbf{A})}\right)-\varrho^{(\beta, \omega, \vartheta, \lambda)}\left(W_{t_{0}}^{(\omega, \vartheta, \mathbf{A})}\right) .
$$

Note that $\mathfrak{I}_{\mathrm{p}}^{(\omega, \mathbf{A})}$ exists at all times because the total delivered energy equals

$$
\begin{aligned}
\lim _{L \rightarrow \infty}\left\{\rho_{t}^{(\beta, \omega, \vartheta, \lambda, \mathbf{A})}\left(H_{L}^{(\omega, \vartheta, \lambda)}+W_{t}^{(\omega, \vartheta, \mathbf{A})}\right)-\varrho^{(\beta, \omega, \vartheta, \lambda)}\right. & \left.\left(H_{L}^{(\omega, \vartheta, \lambda)}\right)\right\} \\
& =\mathfrak{I}_{\mathrm{p}}^{(\omega, \mathbf{A})}(t)+\mathfrak{I}_{\mathrm{d}}^{(\omega, \mathbf{A})}(t)
\end{aligned}
$$


for any $\beta \in \mathbb{R}^{+}, \omega \in \Omega, \vartheta, \lambda \in \mathbb{R}_{0}^{+}, \mathbf{A} \in \mathbf{C}_{0}^{\infty}$ and times $t \geq t_{0}$. See (25) and Theorem 3.2 (ii).

The term $\mathfrak{J}_{\mathrm{p}}^{(\omega, \mathbf{A})}$ is the part of electromagnetic work implying a change of the internal state of the system. By contrast, the second one $\mathfrak{I}_{\mathrm{d}}^{(\omega, \mathbf{A})}$ is the electromagnetic potential energy of the fermion system in the thermal equilibrium state. These two energy increments are directly related to paramagnetic and diamagnetic currents, respectively. For more details, see discussions in [BPH2, Sections 4.3-4.4].

To present this, it is convenient to define the subset

$$
\mathfrak{K}:=\left\{\mathbf{x}=\left(x^{(1)}, x^{(2)}\right) \in \mathfrak{L}^{2}:\left|x^{(1)}-x^{(2)}\right|=1\right\}
$$

of oriented bonds (cf. (1)). Then, by Theorem 3.7, for each $l, \beta \in \mathbb{R}^{+}, \omega \in \Omega$, $\vartheta, \lambda \in \mathbb{R}_{0}^{+}$and any electromagnetic potential $\mathbf{A} \in \mathbf{C}_{0}^{\infty}$, the electric field in its integrated form $\mathbf{E}_{t}^{\eta \mathbf{A}_{l}}$ (cf. (3)-(4) and (36)) implies paramagnetic and diamagnetic currents with linear coefficients being respectively equal to

$$
\begin{aligned}
J_{\mathrm{p}, l}^{(\omega, \mathbf{A})}(t, \mathbf{x}) & :=\frac{1}{2} \int_{t_{0}}^{t} \sum_{\mathbf{y} \in \mathfrak{K}} \sigma_{\mathrm{p}}^{(\omega)}(\mathbf{x}, \mathbf{y}, t-s) \mathbf{E}_{s}^{\mathbf{A}_{l}}(\mathbf{y}) \mathrm{d} s, \\
J_{\mathrm{d}, l}^{(\omega, \mathbf{A})}(t, \mathbf{x}) & :=\int_{t_{0}}^{t} \sigma_{\mathrm{d}}^{(\omega)}(\mathbf{x}) \mathbf{E}_{s}^{\mathbf{A}_{l}}(\mathbf{x}) \mathrm{d} s
\end{aligned}
$$

at any bond $\mathbf{x} \in \mathfrak{K}$ and time $t \geq t_{0}$. Recall that $\sigma_{\mathrm{p}}^{(\omega)}$ and $\sigma_{\mathrm{d}}^{(\omega)}$ are the microscopic charge transport coefficients defined by (42)-(43). Like [BPH2, Theorem 4.1], the following holds:

\section{Theorem 3.8 (Microscopic Joule's law)}

Assume (10)-(11) and (24). Let $l, \beta \in \mathbb{R}^{+}, \omega \in \Omega, \vartheta_{0}, \lambda \in \mathbb{R}_{0}^{+}, \vartheta \in\left[0, \vartheta_{0}\right]$, $\eta \in \mathbb{R}, \mathbf{A} \in \mathbf{C}_{0}^{\infty}$ and $t \geq t_{0}$.

(p) Paramagnetic energy increment:

$$
\mathfrak{I}_{\mathrm{p}}^{\left(\omega, \eta \mathbf{A}_{l}\right)}(t)=\frac{\eta^{2}}{2} \int_{t_{0}}^{t} \sum_{\mathbf{x} \in \mathfrak{K}} J_{\mathrm{p}, l}^{(\omega, \mathbf{A})}(s, \mathbf{x}) \mathbf{E}_{s}^{\mathbf{A}_{l}}(\mathbf{x}) \mathrm{d} s+\mathcal{O}\left(\eta^{3} l^{d}\right) .
$$

(d) Diamagnetic energy:

$$
\begin{aligned}
\mathfrak{I}_{\mathrm{d}}^{\left(\omega, \eta \mathbf{A}_{l}\right)}(t)= & -\frac{\eta}{2} \sum_{\mathbf{x} \in \mathfrak{K}} \varrho^{(\beta, \omega, \vartheta, \lambda)}\left(I_{\mathbf{x}}^{(\omega, \vartheta)}\right)\left(\int_{t_{0}}^{t} \mathbf{E}_{s}^{\mathbf{A}_{l}}(\mathbf{x}) \mathrm{d} s\right) \\
& +\frac{\eta^{2}}{4} \sum_{\mathbf{x} \in \mathfrak{K}} J_{\mathrm{d}, l}^{(\omega, \mathbf{A})}(t, \mathbf{x}) \int_{t_{0}}^{t} \mathbf{E}_{s}^{\mathbf{A}_{l}}(\mathbf{x}) \mathrm{d} s+\mathcal{O}\left(\eta^{3} l^{d}\right) .
\end{aligned}
$$


(Q) Heat production - Internal energy increment:

$$
\begin{aligned}
\mathbf{S}^{\left(\omega, \eta \mathbf{A}_{l}\right)}(t)= & -\frac{\eta^{2}}{2} \sum_{\mathbf{x} \in \mathfrak{K}} J_{\mathrm{p}, l}^{(\omega, \mathbf{A})}(t, \mathbf{x})\left(\int_{t_{0}}^{t} \mathbf{E}_{s}^{\mathbf{A}_{l}}(\mathbf{x}) \mathrm{d} s\right) \\
& +\mathfrak{I}_{\mathrm{p}}^{\left(\omega, \eta \mathbf{A}_{l}\right)}(t)+\mathcal{O}\left(\eta^{3} l^{d}\right) .
\end{aligned}
$$

(P) Electromagnetic potential energy:

$$
\begin{aligned}
\mathbf{P}^{\left(\omega, \eta \mathbf{A}_{l}\right)}(t)= & \frac{\eta^{2}}{2} \sum_{\mathbf{x} \in \mathfrak{K}} J_{\mathrm{p}, l}^{(\omega, \mathbf{A})}(t, \mathbf{x})\left(\int_{t_{0}}^{t} \mathbf{E}_{s}^{\mathbf{A}_{l}}(\mathbf{x}) \mathrm{d} s\right) \\
& +\mathfrak{I}_{\mathrm{d}}^{\left(\omega, \eta \mathbf{A}_{l}\right)}(t)+\mathcal{O}\left(\eta^{3} l^{d}\right) .
\end{aligned}
$$

The correction terms of order $\mathcal{O}\left(\eta^{3} l^{d}\right)$ in assertions $(p),(d),(\boldsymbol{Q})$ and $(\boldsymbol{P})$ are uniformly bounded in $\beta \in \mathbb{R}^{+}, \omega \in \Omega, \vartheta \in\left[0, \vartheta_{0}\right], \lambda \in \mathbb{R}_{0}^{+}$and $t \geq t_{0}$.

Proof: Up to trivial modification taking into account the support of $\mathbf{A}$, the assertions are basically direct consequences of Theorem 3.2 and [BP3, Theorem 4.8], because of Conditions (21)-(22) and (37). For more details, we also refer to the proof of [BPH2, Theorem 4.1].

The uniformity of the above estimates w.r.t. the size $l \in \mathbb{R}^{+}$of the region where the external electromagnetic field is applied is a pivotal technical step to get in a companion paper [BP4] Joule's law when $l \rightarrow \infty$, i.e., a macroscopic version of the above result.

Acknowledgments: This research is supported by the agency FAPESP under Grant 2013/13215-5 as well as by the Basque Government through the grant IT641-13 and the BERC 2014-2017 program and by the Spanish Ministry of Economy and Competitiveness MINECO: BCAM Severo Ochoa accreditation SEV-2013-0323, MTM2014-53850.

\section{References}

[A1] H. ARAKI, Relative Entropy of States of von Neumann Algebras, Publ. Res. Inst. Math. Sci. Kyoto Univ. 11 (1976) 809-833.

[A2] H. AraKi, Relative Entropy of States of von Neumann Algebras II, Publ. Res. Inst. Math. Sci. Kyoto Univ. 13 (1977) 173-192. 
[AM] H. ARAKI AND H. MORIYA, Equilibrium Statistical Mechanics of Fermion Lattice Systems, Rev. Math. Phys. 15 (2003) 93-198.

[BR2] O. BRATteli AND D.W. Robinson, Operator Algebras and Quantum Statistical Mechanics, Vol. II, 2nd ed. Springer-Verlag, New York, 1996.

[BP1] J.-B. BRU AND W. DE SiQueIRA Pedra, Microscopic Foundations of the Meißner Effect - Thermodynamic Aspects, Rev. Math. Phys. 25 (2013) 1350011-1-66.

[BP2] J.-B. BRU AND W. DE SiqueIRA PEdRA, Microscopic Foundations of Ohm and Joule's Laws - The Relevance of Thermodynamics, to appear in the Proceedings of QMATH12 (2014). Preprint mp_arc 14-26.

[BP3] J.-B. BRU AND W. DE SiQueIRA PEDRA, Lieb-Robinson Bounds for Multi-Commutators and Applications to Response Theory (2014). Preprint mp_arc 14-27.

[BP4] J.-B. BRU And W. De Siqueira Pedra, From the 2nd Law of Thermodynamics to the AC-Conductivity Measure of Interacting Fermions in Disordered Media, Math. Models Methods Appl. Sci. (2015) DOI: 10.1142/S0218202515500566.

[BPH1] J.-B. Bru, W. De Siqueira Pedra And C. Hertling, Heat Production of Non-Interacting Fermions Subjected to Electric Fields, Commun. on Pure and Applied Math. 68(6) (2015) 964-1013. DOI: $10.1002 /$ cpa. 21530

[BPH2] J.-B. Bru, W. De Siqueira Pedra And C. Hertling, Microscopic Conductivity of Lattice Fermions at Equilibrium - Part I: NonInteracting Particles, J. Math. Phys. 56 (2015), 051901-1-51

[BPH3] J.-B. Bru, W. De Siqueira Pedra And C. Hertling, ACConductivity Measure from Heat Production of Free Fermions in Disordered Media. To appear in Archive for Rational Mechanics and Analysis (2015). DOI: 10.1007/s00205-015-0935-1

[BPH4] J.-B. Bru, W. De Siqueira Pedra And C. Hertling, Macroscopic Conductivity of Free Fermions in Disordered Media, Rev. Math. Phys. 26(5) (2014) 1450008-1-25. 
[F] D. K. FERry, Ohm's Law in a Quantum World, Science 335(6064) (2012) 45-46.

[GV] G.F. GiUliani And G. Vignale, Quantum Theory of the Electron Liquid, Cambrigde Univ. Press. 2005.

[JP] V. JaksiC AND C.-A. Pillet, A Note on the Entropy Production Formula, Contemp. Math. 327 (2003) 175-181.

[KLM] A. Klein, O. Lenoble, AND P. MülleR, On Mott's formula for the ac-conductivity in the Anderson model, Annals of Mathematics 166 (2007) 549-577.

[KM1] A. KleIN AND P. Müller, The Conductivity Measure for the Anderson Model, Journal of Mathematical Physics, Analysis, Geometry 4 (2008) 128-150.

[KM2] A. KLEIN AND P. MÜLLER, AC-conductivity and Electromagnetic Energy Absorption for the Anderson Model in Linear Response Theory, to appear in Markov processes and Related Fields (2014).

[NOS] B. Nachtergaele, Y. Ogata And R. Sims, Propagation of Correlations in Quantum Lattice Systems, J. Stat. Phys., 124(1) (2006) 1-13.

[NS] B. Nachtergaele And R. Sims, Lieb-Robinson Bounds in Quantum Many-Body Physics, Contemporary Mathematics 529 (2010) 141-176.

[PW] W. Pusz And S. L. Woronowicz, Passive States and KMS States for General Quantum Systems, Commun. math. Phys. 58 (1978) 273-290.

[Si] R. Sims, Lieb-Robinson bounds and quasi-locality for the dynamics of many-body quantum systems. Mathematical Results in Quantum Physics. Proceedings of the QMath 11 Conference in Hradec Kralove, Czech Republic 2010, 95 - 106, Edited by Pavel Exner. World Scientific, Hackensack, NJ (2011).

[W] B. Weber ET AL., Ohm's Law Survives to the Atomic Scale, Science 335(6064) (2012) 64-67. 